ESAIM: COCV 27 (2021) S8

https://doi.org/10.1051/cocv/2020043
ESAIM: Control, Optimisation and Calculus of Variations

www.esaim-cocv.org

\title{
STABILIZATION OF THE RESPONSE OF CYCLICALLY LOADED LATTICE SPRING MODELS WITH PLASTICITY*
}

\author{
Ivan Gudoshnikov And OLEG MAKARENKOV**
}

\begin{abstract}
This paper develops an analytic framework to design both stress-controlled and displacement-controlled $T$-periodic loadings which make the quasistatic evolution of a one-dimensional network of elastoplastic springs converging to a unique periodic regime. The solution of such an evolution problem is a function $t \mapsto(e(t), p(t))$, where $e_{i}(t)$ is the elastic elongation and $p_{i}(t)$ is the relaxed length of spring $i$, defined on $\left[t_{0}, \infty\right)$ by the initial condition $\left(e\left(t_{0}\right), p\left(t_{0}\right)\right)$. After we rigorously convert the problem into a Moreau sweeping process with a moving polyhedron $C(t)$ in a vector space $E$ of dimension $d$, it becomes natural to expect (based on a result by Krejci) that the elastic component $t \mapsto e(t)$ always converges to a $T$-periodic function as $t \rightarrow \infty$. The achievement of this paper is in spotting a class of loadings where the Krejci's limit doesn't depend on the initial condition $\left(e\left(t_{0}\right), p\left(t_{0}\right)\right)$ and so all the trajectories approach the same $T$-periodic regime. The proposed class of sweeping processes is the one for which the normals of any $d$ different facets of the moving polyhedron $C(t)$ are linearly independent. We further link this geometric condition to mechanical properties of the given network of springs. We discover that the normals of any $d$ different facets of the moving polyhedron $C(t)$ are linearly independent, if the number of displacement-controlled loadings is two less the number of nodes of the given network of springs and when the magnitude of the stress-controlled loading is sufficiently large (but admissible). The result can be viewed as an analogue of the high-gain control method for elastoplastic systems. In continuum theory of plasticity, the respective result is known as Frederick-Armstrong theorem.
\end{abstract}

Mathematics Subject Classification. 37J25, 49K24, 74C05.

Received March 24, 2019. Accepted July 8, 2020.

\section{INTRODUCTION}

The classical theory of elastoplasticity offers comprehensive results, commonly known as shakedown theorems, about the maximal magnitude of the applied loading (shakedown load limit) beyond which the response of elastoplastic material necessarily involves plastic deformation regardless of the initial distribution of stresses in the material, see Section 10 in [31]. In other words, shakedown theorems measure the distance between the current stress distribution in the material to a certain boundary (called yield surface) built of the spatially

* The study was funded by the National Science Foundation (grant CMMI-1916876). The authors are grateful to anonymous referees, whose very useful comments helped to improve the paper.

Keywords and phrases: Elastoplasticity, Moreau's sweeping process, quasistatic evolution, periodic loading, stabilization.

Department of Mathematical Sciences, The University of Texas at Dallas, Richardson, USA.

** Corresponding author: makarenkov@utdallas .edu 
distributed elastic limits. The fundamental result by Frederick and Armstrong [19] says that, if the amplitude of a $T$-periodic loading exceeds the shakedown limit, then the stress distribution asymptotically approaches a unique $T$-periodic steady cycle which doesn't depend on the initial stress distribution (uniqueness of the response). Frederick-Armstrong highlight that convergence to a unique cyclic state is guaranteed when the yield surface contains no lines of zero curvature ([19], p. 159). Assuming this or another restriction on the geometry of the yielding surface (such as von Mises, Tresca, or Mohr-Coulomb criteria), many authors computed the steady cycle by discretizing the problem spatially [21, 24, 41] and/or temporarily [47, 48, 56], and by solving the associated minimization problems for the successive discrete states. For the convergence of the discretized problem as the mesh gets smaller, see Crismale [18], Jakabcin [30], and references therein. Applications included the performance of various structures and metal matrix composites under cyclic loadings, see $[28,54]$.

Aiming to design materials with better properties, there has been a great deal of work lately where a discrete structure comes not from an associated model of continuum mechanics, but from a certain microstructure formulated through a lattice of elastic springs [5, 42] (metals), [27, 55] (polymers), [14] (titanium alloys), $[32,51,52]$ (biological materials). Despite of the fact that fatigue crack initialization in heterogeneous materials strongly depends on local micro-plasticity (see e.g. Blechman [6]), the current literature features only numeric results about the dynamics of the lattices of elastoplastic springs. Important papers in this direction are e.g. Buxton et al. [13] and Chen et al. [15].

The goal of the present paper is to initiate the development of a qualitative theory of the lattices of elastoplastic springs and to offer an analogue of the Frederick-Armstrong theorem for such systems.

We stick to the setting of ideal plasticity (the stress of each spring is constrained within so-called elastic limits beyond which plastic deformation begins) and investigate the asymptotic distribution of the stresses $s(t)=\left(s_{1}(t), \ldots, s_{m}(t)\right)$ of a network of $m$ elastoplastic springs. Starting with a graph of $m$ connected elastoplastic springs, the paper takes Moreau's approach [45] to write down the equation for stress $s_{i}$ of spring $i$ without any knowledge about plastic deformation of the spring and relying entirely on the geometry of the graph and elastic limits $\left[c_{i}^{-}, c_{i}^{+}\right]$of the springs. The plasticity is accounted through the $m$-dimensional parallelepipedshaped constraint $C(t)$, whose boundary can be viewed as discretized yield surface, see Figure 3. Beneficially for the performance of computational routines, Moreau concluded that the stress-vector $s(t)=\left(s_{1}(t), \ldots, s_{m}(t)\right)$ of springs is confined within a time-independent low-dimensional hyperplane $V$. It is also due to Moreau that external time-varying loadings enter the equations of dynamics through a time-varying vector $c(t)$ that acts as displacement of the parallelepiped $C$ (Fig. 3). The only obstacle towards practical implementation of the Moreau approach [45] in the context of spring network modeling is that [45] deals with abstract configuration spaces. This paper clears this obstacle and fully adapts Moreau sweeping process framework to the modeling of networks of elastoplastic springs.

After a suitable change of variables that we rigorously incorporate in the next section of the paper, the equations of Moreau (Moreau's sweeping process) can be formulated as

$$
-y^{\prime}(t) \in N_{(C+c(t)) \cap V}(y(t)), \quad y(t) \in \mathbb{R}^{m},
$$

where

$$
N_{\mathcal{C}}(x)= \begin{cases}\left\{\zeta \in \mathbb{R}^{n}:\langle\zeta, c-x\rangle \leqslant 0, \text { for any } c \in \mathcal{C}\right\}, & \text { if } x \in \mathcal{C}, \\ \emptyset, & \text { if } x \notin \mathcal{C}\end{cases}
$$

is a normal cone to the set $\mathcal{C}$ at point $x$ and $V$ is a subspace of $\mathbb{R}^{m}$. For Lipschitz-continuous $t \mapsto c(t)$ (which we show to be the case when the external loading is Lipschitz-continuous) sweeping process (1.1) possesses usual properties of the existence and continuous dependence of solutions on the initial conditions, see e.g. Kunze and Monteiro Marques [38] (the interested reader can e.g. consult Adly et al. [2] and Recupero [49] for the existence of solutions under more relaxed assumptions about the regularity of $C(\cdot))$. 
An alternative approach that uses sweeping process for studying evolution of networks of elastoplastic springs is offered in Bastien et al. [4] (see also Brogliato [9], Sect. 2.3). The approach of [4] leads to a sweeping process with a parallelepipedal constraint while the constraint $(C+c(t)) \cap V$ can be a simplex (even though $C+c(t)$ is a parallelepiped), which shape is in the heart of our stabilization analysis. We discuss this in Remark 3.6 in more details.

When sweeping process (1.1) includes a vector field on top of the normal cone (so-called perturbed sweeping process), multiple results are available to stabilize the dynamics of a sweeping process. Important results in this direction are obtained in Leine and van de Wouw [39, 40], Brogliato [8], and Brogliato-Heemels [10], Kamenskiy et al. [33].

As for the regular sweeping process (1.1), very limited tools to control the asymptotic response are currently available (in contrast to optimal control results developed e.g. in Colombo et al. [16]). The asymptotic behavior of sweeping process (1.1) with $T$-periodic excitation $t \mapsto c(t)$ was studied in Krejci [36], who proved the convergence of solutions of (1.1) to a $T$-periodic attractor in the case $V=\mathbb{R}^{m}$, i.e. $(C+c(t)) \cap V=C+c(t)$. If sweeping process (1.1) decomposes into a Cartesian product of several sweeping processes (1.1) with $m=1$, the global asymptotic stability can be concluded from the theory of Prandtl-Ishlinskii operators (Brokate-Sprekels [12], Krasnosel'skii-Pokrovskii [35], Visintin [53]). In the case of an arbitrary $T$-periodic polyhedron $t \mapsto(C+c(t)) \cap$ $V$, it looks possible to follow the ideas of Adly et al. [1] and obtain global asymptotic stability of a periodic solution by assuming that $g(t)$ lies strictly inside the normal cone $N_{(C+c(t)) \cap V}(y)$ for at least one $y$ and $t \in[0, T]$. The present paper takes a different route and establishes convergence of solutions to a unique $T$-periodic regime in terms of the shape of the moving constraint $(C+c(t)) \cap V$ only.

The paper is organized as follows. The next section rigorously formulates the system of laws of quasistatic evolution for a one-dimensional network of $m$ elastoplastic springs on $n$ nodes. In Section 3 we construct the vector $c(t)$ and the hyperplane $V$ for arbitrary networks of elastoplastic springs of 1-dimensional nodes. We discover that the functions $g(t) \in V$ and $h(t) \perp V$ in the orthogonal decomposition of $c(t)$ (see Fig. 3) correspond to displacement-controlled and stress-controlled loadings respectively (as termed in [28]). The achievement of Section 2 makes it possible to link the dynamics of networks of elastoplastic springs to the dynamics of sweeping processes.

In Section 4 we consider a general sweeping process with a moving set of a form $\cap_{j=1}^{M}\left(C_{j}+c_{j}(t)\right)$, where $C_{j}$ are closed convex sets, and prove (Thm. 4.3) the convergence of all solutions to a $T$-periodic attractor $X(t)$. Section 4.2 (Thm. 4.8) sharpens the conclusion of Theorem 4.3 for the case when $\cap_{j=1}^{M}\left(C_{j}+c_{j}(t)\right)$ is the polyhedron $\Pi(t) \cap V$. Theorem 4.8 shows that even though $X(t)$ may consist of a family of functions, all those functions exhibit certain similar dynamics. Specifically, we prove that any two function $x_{1}, x_{2} \in X$ reach (leave) any of the facets of $\Pi(t) \cap V$ at the same time. Section 4.3 (Thm. 4.10) reformulates the conclusion of Theorem 4.8 in terms of the sweeping process of a network of elastoplastic springs.

Section 5 introduces a class of networks of elastoplastic springs whose stresses converge to a unique $T$-periodic regime regardless of applied $T$-periodic loadings as long as the magnitudes of those loadings are sufficiently large. We begin Section 5 by addressing a general sweeping process in a vector space $E$ of dimension $d$ with a $T$ periodic polyhedral moving set with no connection to networks of springs. Theorem 5.1 of Section 5.1 states that the periodic attractor of such a sweeping process contains at most one non-constant solution, if normals of any $d$ different facets of the moving polyhedron $C(t)$ are linearly independent. Section 5.2 is the main achievement of this paper, where we introduce a class of networks of elastoplastic springs for which the condition of Theorem 5.1 can be easily expressed in terms of the magnitudes of the periodic loadings. We discovered (Thm. 5.4) that global stability of a unique periodic regime occurs when both displacement-controlled and stress-controlled loadings are large enough. A conclusions sections concludes the paper.

Appendixes A-D follow the main text of the paper. In Appendix A we clarify how our system (2.1)-(2.5) for quasi-static evolution of the elastic elongations and relaxed lengths of springs can be obtained from the abstract framework by Moreau [45]. Appendix B quotes some standard results from graph theory, linear algebra, and convex analysis, that are used in the paper. Appendix C is devoted to the proof of the above-mentioned Krejci convergence theorem (following [36]). Finally, Appendix D includes a figure that illustrates the structure of configuration space $\mathbb{R}^{m}$ and the construction of the variables used in Theorem 3.1. 


\section{LAWS OF QUASISTATIC EVOLUTION FOR ONE-DIMENSIONAL NETWORKS OF ELASTOPLASTIC SPRINGS}

We consider a one-dimensional network of $m$ elastoplastic springs of lengths $e_{j}+p_{j}, j \in \overline{1, m}$, where $e_{j}$ is elastic elongation and $p_{j}$ is the relaxed length respectively. Spring $j$ is elastoplastic in the sense that the relaxed length $p_{j}$ changes when the stress of the spring attempts to exceed certain bounds. The bounds of the stress of spring $j$ are denoted by $\left[c_{j}^{-}, c_{j}^{+}\right]$and $a_{j}$ stays for the Hooke's coefficient of this spring. Each spring connects two of $n$ nodes according to $e_{j}+p_{j}=\xi_{\psi_{j}}-\xi_{\phi_{j}}$, where $\phi_{j}$ and $\psi_{j}$ are the indices of the left and right nodes of spring $j$ respectively and $\xi_{i}$ is the position of node $i$. So defined, the one-dimensional network of springs is an oriented graph on $n$ nodes, where spring $j$ is a directed edge of the graph that points from node $\phi_{j}$ to node $\psi_{j}$ (i.e. always points to the right).

The paper investigates the evolution of the stresses under the influence of two types of loadings being displacement-controlled loading and stress-controlled loading.

\subsection{Displacement-controlled loading}

Displacement-controlled loading $l_{j}(t)$ locks the distance between nodes $\Phi_{k}$ and $\Psi_{k}$ through $k \in \overline{1, q}$ according to the rule

$$
\xi_{\Psi_{k}}-\xi_{\Phi_{k}}=l_{k}(t)
$$

Since we will work with connected graphs of springs only, we assume that for each displacement-controlled loading $k \in \overline{1, q}$, there exists a directed path of springs which connects the left node $\Phi_{k}$ of constraint $k$ with its right node $\Psi_{k}$. Assume that spring $j$ belongs to the path. We say that spring $j$ agrees with the direction of the path, if one passes node $\phi_{j}$ before passing node $\psi_{j}$, when following the path in positive direction. And we say that spring $j$ disagrees with the direction of the path, if one passes node $\psi_{j}$ before passing node $\phi_{j}$, when following the path in positive direction. With each displacement-controlled loading $k$ we can, therefore, associate a so-called incidence vector $R^{k} \in \mathbb{R}^{m}$ whose $j$-th component $R_{j}^{k}$ is $-1,0$, or 1 , according to whether spring $j$ belongs to the path and disagrees with the direction of the path, doesn't belong to the path, or belongs to the path and agrees with the direction of the path, see Figure 1 (we refer the reader to Appendix B for an analytic definition based on Bapat[3], p. 57).

Since the length of spring $j$ equals $e_{j}+p_{j}$, we can use the incidence vector $R^{k}$ to write the following equation of displacement-controlled loading which takes into account the given length $l_{k}(t)$ :

$$
\left(R^{k}\right)^{T}(e+p)=l_{k}(t)
$$

Note, the constant $R_{j}^{k}$ is computed based on the order in which nodes $\phi_{j}$ and $\psi_{j}$ are encountered when following some path of springs from node $\Phi_{k}$ to node $\Psi_{k}$. Since this order doesn't depend on the direction of the $\xi$-axis, we conclude that the value of $R_{j}^{k}$ doesn't depend on the direction of the $\xi$-axis either.

\subsection{Stress-controlled loading and quasi-static balance}

Stress-controlled loading $f_{i}(t)$ models external forces applied at node $i$. The resultant force at node $i$ is a sum of 3 forces:

(a) the resultant of the stresses of all springs applied at node $i$,

(b) the resultant force of all displacement-controlled loadings applied at node $i$,

(c) the stress-controlled loading $f_{i}(t)$.

In contrast with Section 2.1, the constructions of the current section do depend on the direction of the $\xi$-axis. In what follows, saying "to the right (respectively left)" means "in the positive (respectively negative) direction 


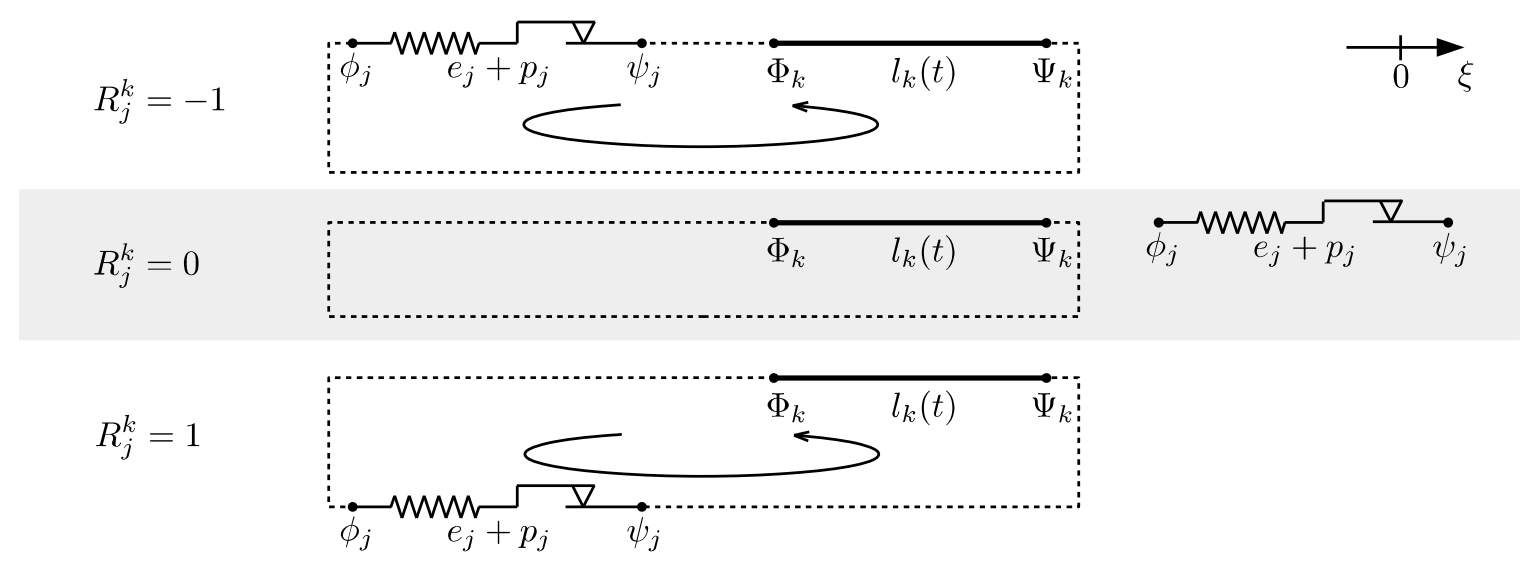

FIGURE 1. Illustration of the signs of the components of the incidence vector $R^{k} \in \mathbb{R}^{m}$. The dotted contour stays for the directed path of springs associated with the vector $R^{k}$. This figure also illustrates that, by construction, the orientation of the cycle obtained by replacing $l_{k}(t)$ by a virtual spring on nodes $\Phi_{k}<\Psi_{k}$ (used in the proof of Thm. 3.1) always disagrees with the orientation of the virtual spring (which by our convention points from node $\Phi_{k}$ to node $\Psi_{k}$, see the beginning of Sect. 2). In what follows, we call the axis of spatial dimension directed from left to right a " $\xi$-axis".

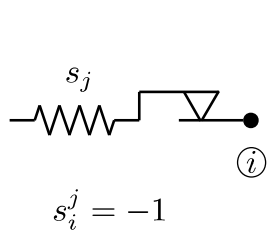

(a)

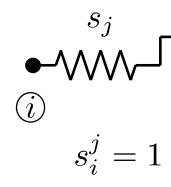

(a)

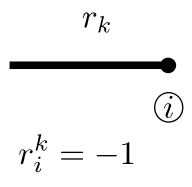

(b)

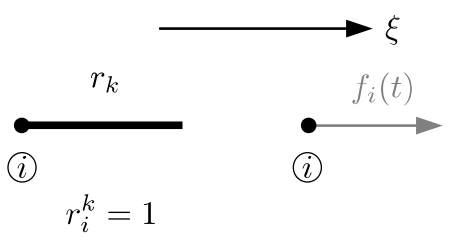

(b) (c)

Figure 2. The 3 possible types of forces applied at node $i$ : (a) elastoplastic spring number $j$, (b) displacement-controlled loading number $k$, and (c) stress-controlled loading number $i$. The figure also illustrates the definition of $s_{i}^{j}$ and $r_{i}^{k}$ that we use to write down the equation of quasi-static balance. The circled " $i$ " means "node $i$ " in the figure. Networks of elastoplastic systems combine several of the 5 configurations mentioned at one node, see e.g. Figure 6 below.

of the $\xi$-axis". The force $f_{i}(t)$ is drawn pointing to the right or to the left according to whether the sign of $f_{i}(t)$ is positive or negative. In particular, the force $f_{i}(t)$ drawn in Figure 2 is of positive sign.

To compute the resultant of the forces of springs applied at nodes, we introduce $n$-dimensional vectors $s^{j}=\left(s_{1}^{j}, \ldots s_{n}^{j}\right)^{T}, j \in \overline{1, m}$, where $s_{i}^{j}=-1, s_{i}^{j}=0$, or $s_{i}^{j}=1$, according to whether spring $j$ is applied to the left from node $i$, not applied to node $i$, or applied to the right from node $i$. Recalling that the stresses of the springs are $s_{1}, \ldots, s_{m}$, the resultant force of all springs applied at node $i$ reads as

$$
s_{1} s_{i}^{1}+\ldots+s_{m} s_{i}^{m} .
$$

Similarly, to compute the resultant force of the displacement-controlled loadings at nodes, we introduce the $n$-dimensional vectors $r^{k}=\left(r_{1}^{k}, \ldots r_{n}^{k}\right)^{T}, k \in \overline{1, q}$, where $r_{i}^{k}=-1, r_{i}^{k}=0$, or $r_{i}^{k}=1$, according to whether the displacement-controlled loading $k$ is applied to the left from node $i$, not applied to node $i$, or applied to the right from node $i$ (see Fig. 2). Denoting by $r_{k}$ the reaction of the displacement-controlled loading $k$, the resultant force of all displacement-controlled loadings applied at node $i$ can be computed as

$$
r_{1} r_{i}^{1}+\ldots+r_{q} r_{i}^{q}
$$


Therefore, the assumption of quasi-static balance at node $i$ reads as

$$
s_{1} s_{i}^{1}+\ldots+s_{m} s_{i}^{m}+r_{1} r_{i}^{1}+\ldots+r_{q} r_{i}^{q}+f_{i}(t)=0
$$

or, in the vector form, for all nodes simultaneously

$$
s_{1} s^{1}+\ldots+s_{m} s^{m}+r_{1} r^{1}+\ldots+r_{q} r^{q}+f(t)=0 .
$$

\subsection{The combined system for the evolution of elastic elongations and relaxed lengths}

With the notations introduced the quasistatic evolution of the stresses $s_{k}$ of springs and reactions $r_{k}$ of displacement-controlled loadings can be described by the following variational system (which corresponds to equations (6.1)-(6.6) in the abstract framework by Moreau [45], see Appendix A)

$$
\begin{aligned}
\text { Elastic elongation: } \quad s=A e & \\
\text { Relaxed length (plastic deformation): } & \dot{p} \in N_{C}(s), \\
\text { Geometric constraint: } & e+p \in D \mathbb{R}^{n}, \quad D \mathbb{R}^{n}=\left\{D \xi: \xi \in \mathbb{R}^{n}\right\} \\
\text { Displacement-controlled loading: } & R^{T}(e+p)=l(t), \\
\text { Static balance under stress-controlled loading: } & s_{1} s^{1}+\ldots+s_{m} s^{m}+r_{1} r^{1}+\ldots+r_{q} r^{q}+f(t)=0,
\end{aligned}
$$

where

$$
\begin{gathered}
s=\left(s_{1}, \ldots s_{m}\right)^{T} \text { - stresses of springs, } \\
r=\left(r_{1}, \ldots r_{q}\right)^{T} \text { - reactions of displacement-controlled loadings, } \\
e=\left(e_{1}, \ldots e_{m}\right)^{T} \text { - elastic elongations of springs, } \\
p=\left(p_{1}, \ldots p_{m}\right)^{T} \text { - relaxed lengths of springs, } \\
l(t)=\left(l_{1}(t), \ldots, l_{q}(t)\right)^{T} \text { - enforced lengths between the nodes } \Phi_{k} \text { and } \Psi_{k}, k \in \overline{1, q}, \\
f(t)=\left(f_{1}(t), \ldots, f_{n}(t)\right)^{T} \text { - stress-controlled loadings acting at nodes } 1, \ldots, n, \\
A=\operatorname{diag}\left(a_{1}, \ldots, a_{m}\right) \text { - matrix of Hooke's coefficients, } a_{j}>0, j \in \overline{1, m}, \\
\quad C=\prod_{j=1}^{m}\left[c_{j}^{-}, c_{j}^{+}\right]-\text {set of vectors within bounds } c_{j}^{-} \leqslant c_{j}^{+}, j \in \overline{1, m}, i . e . \text { admissible stress values, } \\
N_{C}(s)=\prod_{j=1}^{m} N_{\left[c_{j}^{-}, c_{j}^{+}\right]}(s) \text { - the normal cone to } C \text { at } s, \\
\left.D \xi=\left(\xi_{\psi_{j}}-\xi_{\phi_{j}}\right)_{j=1}^{m}-\text { a linear map (represented by an } m \times n \text {-matrix } D \text { of elements from }\{-1,0,1\}\right) \\
\quad \text { that defines the graph of springs, } \\
\left.R=\left(R^{1}, \ldots, R^{q}\right)-m \times q \text {-matrix of the incidence vectors (of elements from }\{-1,0,1\}\right) \\
\quad \text { of displacement-controlled loadings. }
\end{gathered}
$$

Our construction of equations (2.1)-(2.5) above is inspired by the ideas of Moreau ([45], Sect. 3) and follows some of Moreau's constructions (specifically, the definition of matrix $D$ and quasi-static balance (2.5)), who considered so-called lattices of "bars". Moreau discusses different types of bars in Section 3 from [45], but not elastoplastic springs. Plasticity is discussed in Section 4 in [45], but in the context of frictional contacts. In 
Section 6 from [45], Moreau setups an abstract framework ([45], formulas (6.1)-(6.6)) that he calls an elastoplastic system and which includes our system (2.1)-(2.5), see Appendix A. In particular, the law of plastic deformation (2.2) is taken from Moreau ([45], Sect. 6, p. 303), see also Han-Reddy ([23], p. 109). Moreau mentions that his abstract framework is applicable when the bars of Section 3 from [45] are elastoplastic springs, but only a short paragraph is devoted to the details of this approach, see Section 6, page 304 from [45]. In particular, understanding that, for the case of lattices of springs, the term $g(t)$ corresponds to displacement-controlled loadings, is one of the findings of the present paper (as far as the modeling side is concerned).

The $m \times n$-matrix $D$ will be termed the kinematic matrix of the one-dimensional network of $m$ springs on $n$ nodes. Note, the matrix $-D^{T}$ is the incidence matrix of the associated oriented graph of $n$ nodes and $m$ edges, see Appendix B.

Following Moreau [45], we term system (2.1)-(2.5) an elastoplastic system.

\section{Casting the elastoplastic system as SWeeping Process}

\subsection{Derivation of the sweeping process}

In order for (2.4) to be solvable in $e+p$ we assume that the displacement-controlled loadings $\left\{l_{k}(t)\right\}_{k=1}^{q}$ are independent in the sense that

$$
\operatorname{rank}\left(D^{T} R\right)=\operatorname{rank}\left(R^{T} D\right)=q
$$

Mechanically, condition (3.1) ensures that the displacement-controlled loadings don't contradict one another. For example, (3.1) rules out the situation where two displacement-controlled loadings connect same pair of nodes. It follows from condition (3.1) that the matrix equation

$$
R^{T} D L=I_{q \times q}
$$

has a $n \times q$ matrix solution $L$. Furthermore, as we show in the proof of Theorem 3.1, in order for equation (2.5) to be solvable in $s \in \mathbb{R}^{m}$ and $r \in \mathbb{R}^{q}$, the function $f(t)$ must satisfy $f(t) \in D^{T} \mathbb{R}^{m}$, see formula (3.23). That is why we assume $f(t)$ to be given as

$$
f(t)=-D^{T} \bar{h}(t)
$$

for a known function $\bar{h}:[0, \infty) \rightarrow \mathbb{R}^{m}$. As we further clarify in Remark 3.5, Assumption (3.3) is equivalent to

$$
f_{1}(t)+\ldots+f_{n}(t)=0 .
$$

Introducing

$$
U=\left\{x \in D \mathbb{R}^{n}: R^{T} x=0\right\}, \quad V=A^{-1} U^{\perp},
$$

where

$$
U^{\perp}=\left\{y \in \mathbb{R}^{m}:\langle x, y\rangle=0, x \in U\right\}
$$

the space $V$ will be the orthogonal complement of the space $U$ in the sense of the scalar product

$$
(u, v)_{A}=\langle u, A v\rangle
$$


Therefore, any element $x \in \mathbb{R}^{m}$ can be uniquely decomposed as

$$
x=P_{U} x+P_{V} x,
$$

where $P_{U}$ and $P_{V}$ are linear (orthogonal in sense of (3.7)) projection maps on $U$ and $V$ respectively. Define

$$
\begin{aligned}
g(t) & =P_{V} D L l(t), \\
h(t) & =P_{U} A^{-1} \bar{h}(t), \\
N_{C}^{A}(x) & = \begin{cases}\left\{\xi \in \mathbb{R}^{m}:\langle\xi, A(c-x)\rangle \leqslant 0, \text { for any } c \in C\right\}, & \text { if } x \in C, \\
\emptyset, & \text { if } x \notin C, \\
\Pi(t) & =A^{-1} C+h(t)-g(t),\end{cases}
\end{aligned}
$$

and consider the following differential inclusions

$$
\begin{aligned}
-\dot{y} & \in N_{\Pi(t) \cap V}^{A}(y), \\
\dot{z} & \in\left(N_{\Pi(t)}^{A}(y)+\dot{y}\right) \cap U,
\end{aligned}
$$

with initial conditions

$$
\begin{aligned}
& y(0) \in \Pi(0) \cap V, \\
& z(0) \in U .
\end{aligned}
$$

The function $g(t)$ will be termed the effective displacement-controlled loading. Similarly, $h(t)$ is termed the effective stress-controlled loading.

In what follows we are going to establish an equivalence between systems (2.1)-(2.5) and (3.11)-(3.14). Solvability of sweeping process (3.11) is discussed in Section 4.1. Solvability of the sweeping process (3.11) that comes from an abstract infinite-dimensional elastoplastic model (rather than from a discretized version modeling a network of springs) is addressed in Crismale [18].

According to Moreau ([45], Prop. of Sect. 6.d), the problem (3.12), (3.14) admits an absolutely continuous (possibly non-unique) solution $z$ on $[0, T]$ for any absolutely continuous solution $y$ of (3.11), (3.13) defined on $[0, T]$. The analysis of the dynamics of the elastic elongation $e(t)$ therefore reduces to the analysis of the solution $y$ of the sweeping process (3.11). In particular, stabilization of (3.11) will imply stabilization of both elastic elongations $e(t)=\left(e_{1}(t), \ldots, e_{m}(t)\right)^{T}$ and stresses $s(t)=\left(s_{1}(t), \ldots, s_{m}(t)\right)^{T}$ of springs.

Theorem 3.1. Let $D$ be the kinematic matrix of a connected network of m elastoplastic springs on $n$ nodes. Let $R$ be a matrix of incidence vectors of $q$ displacement-controlled constraints, which are independent in the sense of (3.1). Assume that stress-controlled loading $t \mapsto h(t)$ and displacement-controlled loading $t \mapsto l(t)$ are both absolutely continuous functions. Assume that the stress-controlled loading doesn't exceed the safe load bounds, i.e.

$$
(C+A h(t)) \cap U^{\perp} \neq \emptyset, \text { for all } t \in[0, T]
$$

holds for $C, U$ and $h$ as defined in (2.2), (3.5), and (3.9). If absolutely continuous functions $(s(t), e(t), p(t), r(t))$ satisfy (2.1)-(2.5) a.e. on [0,T], then the absolutely continuous functions

$$
\begin{aligned}
& y(t)=e(t)+h(t)-g(t), \\
& z(t)=e(t)+p(t)+h(t)-g(t)
\end{aligned}
$$




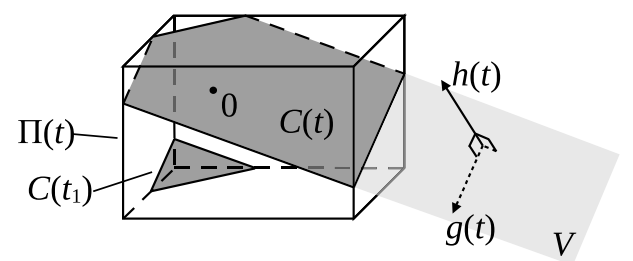

Figure 3. Moving constraint for different values of time. Vectors $g(t) \in V, h(t) \in U$ are orthogonal in the sense of (3.7).

satisfy (3.11)-(3.14) a.e. on $[0, T]$. Conversely, if absolutely continuous functions $(y(t), z(t))$ satisfy (3.11)-(3.14) a.e. on $[0, T]$, then $(e(t), p(t))$ found from (3.16) solve (2.1)-(2.5) a.e. on $[0, T]$ with $s(t)=A e(t)$ and with some suitable $r(t)$.

We refer the reader to Han-Reddy [23] for formulations of the safe load condition (3.15) in the context of classical (continuum) theory of plasticity.

Remark 3.2. Since $P_{U} g(t)=0$, assuming (3.15) is equivalent to assuming $\Pi(t) \cap V \neq \emptyset, t \in[0, T]$.

Remark 3.3. Since $0 \in U^{\perp}$, condition (3.15) always holds when $h(t) \equiv 0$ and $0 \in C$. Geometrically, condition (3.15) means that the parallelepiped $\Pi(t)$ and the hyperplane $V$ in Figure 3 do intersect. Mechanically, condition (3.15) accounts for the fact that the stresses of the elastoplastic springs are bounded and cannot balance arbitrary large stress-controlled loadings.

Remark 3.4. The function $g(t)$ and the matrix $P_{V} D L$ don't depend on the choice of matrix $L$. Indeed, let $\tilde{g}(t)$ be the function $g(t)$ obtained by replacing $L$ by $\tilde{L}$. Then using (3.2) and (3.5) we get

$$
0_{q \times q}=R^{T} D(L-\tilde{L})=R^{T}\left(P_{U} D(L-\tilde{L})+P_{V} D(L-\tilde{L})\right)=R^{T} P_{V} D(L-\tilde{L}),
$$

so $P_{V} D(L-\tilde{L}) \mathbb{R}^{q} \subset U$. Therefore, $P_{V} D(L-\tilde{L})=0_{m \times q}$ and $P_{V} D L=P_{V} D \tilde{L}$. The conclusion $g(t)-\tilde{g}(t)=0$ follows from (3.8).

Remark 3.5. Note, the existence of representation (3.3) is equivalent to the validity of (3.4). Indeed, since $\operatorname{dim} \operatorname{Ker} D+\operatorname{rank} D=n$, by the rank-nullity theorem ([20], Thm. 2.3, see also Appendix B) and $\operatorname{rank} D=$ $n-1$ by Bapat ([3], Lem. 2.2) (the rank of the incidence matrix of a connected graph is one less the number of nodes, see also Lem. B.2 in Appendix B), one has $\operatorname{dim} \operatorname{Ker} D=1$. Since, by definition, every row of $D$ contains exactly two nonzero elements 1 and -1 , it holds $D(1, \ldots, 1)^{T}=0$. Therefore, $D^{T} \mathbb{R}^{m}=(\operatorname{Ker} D)^{\perp}=$ $\left\{x \in \mathbb{R}^{n}:(1, \ldots, 1) x=0\right\}$. In other words, if $f(t)=-D^{T} \bar{h}(t)$ for some $\bar{h}(t)$ then $(1, \ldots, 1) f(t)=0$. Conversely, if $(1, \ldots, 1) f(t)=0$, then $f(t) \in D^{T} \mathbb{R}^{m}$, which means the existence of $\bar{h}(t)$ such that $f(t)=-D^{T} \bar{h}(t)$.

Remark 3.6. An alternative framework that associates a sweeping process with the relations (2.1)-(2.5) is offered in Bastien et al. [4]. This alternative approach describes the elastic component $e(t)$ through a differential inclusion of the form

$$
-\dot{\zeta} \in N_{\mathcal{C}+c(t)}(\zeta)+F(\zeta),
$$

where $\mathcal{C}$ is a parallelepiped and $c(t)$ is a single-valued vector-function. Sweeping process (3.17) can be converted to

$$
-\dot{\sigma} \in N_{\mathcal{C}}(\sigma)+G(t, \sigma)
$$


To investigate the existence and uniqueness of solutions to (3.18) Bastien et al. [4] uses the theory of differential inclusions with time-independent maximal monotone operators (specifically [4], Thm. 2.11). The sweeping process (3.11) (coming from the approach by Moreau [45]) cannot be converted to (3.17) or (3.18). At the same time, Theorem 2.11 from [4] can probably be extended to differential inclusions with time-varying maximal monotone operators and applied to the analysis of the existence and uniqueness of solutions to (3.11). Considering a moving constraint of the form $\Pi(t) \cap V$ as opposed to $\mathcal{C}+c(t)$ is essential for the present paper because we achieve stabilization of (3.11) by providing a class of elastoplastic systems where the parallelepiped $\Pi(t)$ reduces to a simplex upon an intersection with $V$ (see Thm. 5.4). As a matter of fact, the parallelepiped $\mathcal{C}+c(t)$ of (3.17) is a simplex only in dimension 1. We stress that for the purpose of mere existence and uniqueness of the elastic elongation $e(t)$ of elastoplastic system (2.1)-(2.5) both Bastien et al. [4] and Moreau [45] approaches work equally well.

Remark 3.7. The work by Brogliato-Thibault [11] may serve as a framework as for how sweeping process (3.11) can be converted into an equivalent complementarity system, which will provide a connection between elastoplastic systems and complementarity problems.

In Appendix D we offer a diagram (Fig. D.1) that illustrate the construction of the spaces $U, V$ and the moving set $\Pi(t) \cap V$.

Proof of Theorem 3.1. The system of (2.3) and (2.4) is equivalent to

$$
e(t)+p(t) \in U^{l}(t), \quad \text { where } \quad U^{l}(t)=\left\{x \in D \mathbb{R}^{n}: R^{T} x=l(t)\right\} .
$$

Applying the both sides of (3.2) to $l(t)$, we get $R^{T} D L l(t)=l(t)$, which implies $D L l(t) \in U^{l}(t)$. Therefore,

$$
U^{l}(t)=U+D L l(t)
$$

and (3.19) can be rewritten as

$$
e(t)+p(t) \in U+D L l(t)
$$

or, equivalently,

$$
e+p \in U+g(t) .
$$

We will now make use of the quasi-static balance (2.5), which is currently formulated at nodes. We will now rewrite it at springs to obtain a new inclusion for the elongations of springs complementary to (3.20).

By the definition of matrix $D$, the $j$ th column of $D^{T}$ has +1 (respectively -1 ) for the line $i$, if $i$ th node is the right (respectively left) endpoint of spring $j$, see the illustration at Figure 4 . On the other hand $s_{i}^{j}=+1$, if node $i$ is the left end of spring $j$ and $s_{i}^{j}=-1$ if node $i$ is the right end of spring $j$, see Figure 2 . Therefore,

$$
s_{1} s^{1}+\ldots+s_{m} s^{m}=-D^{T} s .
$$

We now claim that the resultant force of the displacement-controlled loadings can be rewritten through the matrix $D^{T}$ as follows

$$
r_{1} r^{1}+\ldots+r_{q} r^{q}=-D^{T} R r, \quad \text { where } \quad r=\left(r_{1}, \ldots, r_{q}\right)^{T}
$$

Indeed, by the definition of vector $r^{k} \in \mathbb{R}^{n}$, the matrix $\left(\begin{array}{c}-D \\ \left(r^{k}\right)^{T}\end{array}\right)^{T}$ is the incidence matrix of the oriented graph of springs $s_{1}, \ldots, s_{m}$ on nodes $1, \ldots, n$ supplemented with a virtual spring connecting the nodes of the 

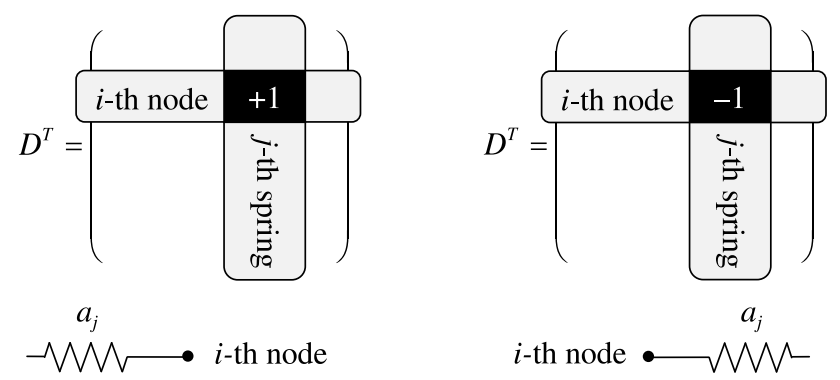

Figure 4. The meaning of the columns and rows of matrix $D^{T}$. The cell equals +1 , if the $i$ th node is the right endpoint for spring $j$. Conversely, the cell equals -1 , if the $i$ th node is the left endpoint for spring $j$.

displacement-controlled loading $k$ (see Appendix B for the definition of incidence matrix). We can now use this virtual spring in order to close the path of springs given by the incidence vector $R^{k}$ and to obtain an oriented cycle whose orientation disagrees with the direction of the virtual spring, see Figure 1 . The incidence vector of this cycle is $\left(\begin{array}{c}R^{k} \\ -1\end{array}\right)$ (see Appendix B for the definition of incidence vector). Therefore, according to Bapat ([3], p. 57) (see also Lem. B.1 in Appendix B), we now have

$$
\left(\begin{array}{c}
-D \\
\left(r^{k}\right)^{T}
\end{array}\right)^{T}\left(\begin{array}{c}
R^{k} \\
-1
\end{array}\right)=0, \quad k \in \overline{1, q}
$$

from which (3.22) follows. Using representations (3.21) and (3.22) the equation of quasi-static balance (2.5) can be rewritten as

$$
-D^{T} s-D^{T} R r+f(t)=0
$$

which proves that the stress-controlled loading $f(t)$ always needs to admit a representation (3.3) for the equation of quasi-static balance (2.5) to hold. Using (3.3) the equation (3.23) further transforms to

$$
-D^{T} s-D^{T} R r-D^{T} \bar{h}(t)=0,
$$

which has a solution $(s(t), r(t))$ if and only if

$$
s(t)+\operatorname{Rr}(t)+\bar{h}(t) \in \operatorname{Ker} D^{T} .
$$

Keeping $s(t)$ fixed, the latter inclusion can be solved for $r(t) \in \mathbb{R}^{q}$ if and only if

$$
s(t)+\bar{h}(t) \in \operatorname{Ker} D^{T}+R \mathbb{R}^{q}=\left(D \mathbb{R}^{n}\right)^{\perp}+\left\{x \in \mathbb{R}^{m}: R^{T} x=0\right\}^{\perp}=\left(D \mathbb{R}^{n} \cap\left\{x \in \mathbb{R}^{m}: R^{T} x=0\right\}\right)^{\perp}=U^{\perp}
$$

see e.g. Friedberg et al. ([20], Ex. 17, p. 367) for the property Ker $D^{T}=\left(D \mathbb{R}^{n}\right)^{\perp}$. If $s(t)$ satisfies (3.25), then by $(3.9)$

$$
\begin{aligned}
s(t)+A \bar{h}(t)= & s(t)+A\left(P_{U} A^{-1} \bar{h}(t)+P_{V} A^{-1} \bar{h}(t)-P_{V} A^{-1} \bar{h}(t)\right) \in \\
& \in s(t)+A A^{-1} \bar{h}(t)+A V=s(t)+\bar{h}(t)+U^{\perp} \in U^{\perp} .
\end{aligned}
$$


Vice versa, if $s(t)$ satisfies (3.26) then

$$
s(t)+\bar{h}(t)=s(t)+A\left(P_{U} A^{-1} \bar{h}(t)+P_{V} A^{-1} \bar{h}(t)\right)=s(t)+A h(t)+A P_{V} A^{-1} \bar{h}(t) \in U^{\perp},
$$

which is (3.25). By applying $A^{-1}$ to (3.26), we get

$$
e+h(t) \in V
$$

In the remainder of the proof we use derivatives of absolutely continuous functions which exist a.e. on $[0, T]$. When using the derivative we implicitly assume that the derivative is being computed at a point of $[0, T]$ where it exists. Since $g(t) \in V$ and $h(t) \in U$ we can rewrite (3.20), (3.27) and (2.2) as

$$
\begin{aligned}
e+p-g(t)+h(t) & \in U, \\
e+h(t)-g(t) & \in V, \\
\dot{p} & \in N_{C}(A e) .
\end{aligned}
$$

Introducing the change of the variables and using the substitutions $p=z-y$ and $e=y-h(t)+g(t)$,

$$
\begin{aligned}
& \dot{z}-\dot{y} \in N_{A^{-1} C}^{A}(y-h(t)+g(t)) \\
& z \in U \\
& y \in V
\end{aligned}
$$

Let $(y, z)$ be a solution of (3.28). Since $z \in U$ we have $-\dot{z} \in-U=U=V^{\perp_{A}}=N_{V}^{A}(y)$, where $V^{\perp_{A}}$ is the orthogonal complement of $V$ in the sense of the scalar product $(\cdot, \cdot)_{A}$, and the inclusion (3.11) computes as follows:

$$
-\dot{y} \in N_{A^{-1} C}^{A}(y-h(t)+g(t))-\dot{z} \in N_{A^{-1} C+h(t)-g(t)}^{A}(y)+N_{V}^{A}(y)=N_{\left(A^{-1} C+h(t)-g(t)\right) \cap V}^{A}(y),
$$

where the last equality holds due to (3.15) (where both intersecting sets are polyhedral, we use Rockafellar [50], Cor. 23.8.1, see also Appendix B). The inclusion (3.12) follows by combining

$$
\dot{z}=\dot{z}-\dot{y}+\dot{y} \in N_{A^{-1} C+h(t)-g(t)}^{A}(y)+\dot{y}
$$

with the property $z(t) \in U$ observed in (3.28). Vice versa, if $(y, z)$ is a solution of (3.11)-(3.12), then

$$
\begin{aligned}
& -y \in V, \\
& \dot{z} \in N_{A^{-1} C+h(t)-g(t)}^{A}(y)+\dot{y}, \\
& \dot{z} \in U,
\end{aligned}
$$

which implies (3.28) when combined with (3.14).

We acknowledge that the ideas of the proof of Theorem 3.1 are due to Moreau [45], who however worked in abstract configuration spaces and only briefly mentions how to relate sweeping process (3.11) to networks of elastoplastic springs (2.1)-(2.5). In Appendix A we show how our system (2.1)-(2.5) can be derived from the Moreau's framework.

Formulas (3.8)-(3.10) establish a connection between mechanical properties of applied loading and geometric properties of the moving constraint $\Pi(t) \cap V$. Specifically, varying the stress-controlled loading $f(t)$ moves $\Pi(t)$ in the direction perpendicular to $V$ in the sense of the scalar product (3.7). In contrast, varying the displacement-controlled loading $l(t)$ moves $\Pi(t)$ in the direction parallel $V$. We also see that the variety of 
possible perpendicular motions coming from $f(t)$ is limited by the dimension of the space $U$, which will be computed in Section 3.3 (Lem. 3.8). The dimension of possible directions for the parallel motion in $V$ is not always $\operatorname{dim} V$, but is related to the rank of matrix $\bar{L}$, which we compute in Section 3.5, see formula (3.46).

\subsection{A polyhedral description of moving sets for elastoplastic systems and reduction to subspace $V$}

In this section we rewrite the moving set $\Pi(t) \cap V$ and sweeping process (3.12) in a slightly different form which is more suitable for further analysis. From

$$
A^{-1} C=\bigcap_{j=1}^{m}\left\{x \in \mathbb{R}^{m}: \frac{1}{a_{j}} c_{j}^{-} \leqslant x_{j} \leqslant \frac{1}{a_{j}} c_{j}^{+}\right\}
$$

we have

$$
A^{-1} C+h(t)-g(t)=\bigcap_{j=1}^{m}\left\{x \in \mathbb{R}^{m}: c_{j}^{-}+a_{j} h_{j}(t) \leqslant\left\langle e_{j}, A x+A g(t)\right\rangle \leqslant c_{j}^{+}+a_{j} h_{j}(t)\right\},
$$

where $e_{j} \in \mathbb{R}^{m}$ is the vector with 1 in the $j$ th component and zeros elsewhere. Since $g(t) \in V$, one has

$$
\left\langle e_{j}, A x+A g(t)\right\rangle=\left\langle P_{U} e_{j}+P_{V} e_{j}, A x+A g(t)\right\rangle=\left\langle P_{V} e_{j}, A x+A g(t)\right\rangle, \quad x \in V,
$$

and we conclude

$$
\Pi(t) \cap V=\bigcap_{j=1}^{m}\left\{x \in V: c_{j}^{-}+a_{j} h_{j}(t) \leqslant\left\langle n_{j}, A x+A g(t)\right\rangle \leqslant c_{j}^{+}+a_{j} h_{j}(t)\right\},
$$

where $n_{j}=P_{V} e_{j}$, i.e. $n_{j}$ are the columns of the projection matrix $P_{V}$.

Furthermore, since $y(t) \in V$ for all $t$ and $y^{\prime}(t) \in V$ for a.a. $t$ we can restrict the normal cone from (3.11) to the normal cone defined within the subspace $V$, which also appears to be the intersection of the original normal cone with $V$ :

$$
N_{\mathcal{C}}^{V}(x):=N_{\mathcal{C}}^{A}(x) \cap V= \begin{cases}\{\xi \in V:\langle\xi, A(c-x)\rangle \leqslant 0, \text { for any } c \in \mathcal{C}\}, & \text { if } x \in \mathcal{C}, \\ \emptyset, & \text { if } x \notin \mathcal{C} .\end{cases}
$$

Therefore we can restrict sweeping process (3.11) to the following one which is defined solely within $V$ :

$$
-\dot{y} \in N_{\Pi(t) \cap V}^{V}(y) .
$$

In the following chapters we are going to analyze the dynamics of sweeping process (3.30) with the moving set in form (3.29).

\subsection{Sweeping processes of particular elastoplastic systems}

In this section we consider a particular network of elastoplastic springs and offer a guideline that can be used to derive the associated sweeping process (3.11) in closed form.

The following lemma will be used to compute the dimension of $U$.

Lemma 3.8. If (3.1) is satisfied, then

$$
\operatorname{dim} U=n-q-1 .
$$




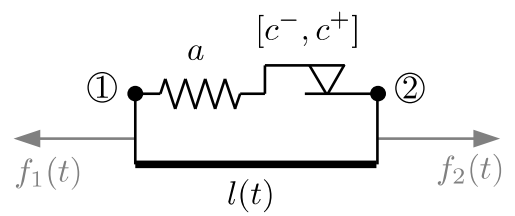

Figure 5. The minimal elastoplastic system with $\operatorname{dim} U=0$.

Proof. Let $E=D \mathbb{R}^{n}$. Viewing $R^{T}$ as a linear map from $E$ to $\mathbb{R}^{q}$ the rank-nullity theorem (Friedberg et al. [20], Thm. 2.3, see also Appendix B) gives

$$
\operatorname{dim} \operatorname{Ker} R^{T}+\operatorname{rank} R^{T}=\operatorname{dim} E,
$$

where $\operatorname{dim} \operatorname{Ker} R^{T}=\operatorname{dim} U$ by (3.5), $\operatorname{rank} R^{T}=q$ by (3.1), and $\operatorname{dim} E=n-1$ by Bapat ([3], Lem. 2.2) (see also Lem. B.2 in Appendix B).

Lemma 3.8 highlights that if the displacement-controlled loadings are independent in the sense of condition (3.1) and if the number $q$ of displacement-controlled loadings is 1 less the number of nodes $n$, then $\operatorname{dim} U=0$ and so the stress-controlled loadings have no influence on the dynamics of the system. The minimal elastoplastic system that illustrates the situation $\operatorname{dim} U=0$ is drawn at Figure 5 (resembling Brogliato [9], p. 258, Ex. 5.3). The stress-controlled loading $l(t)$ locks the dynamics completely and the forces $f_{1}(t)$ and $f_{2}(t)$ have no influence on the system. To construct the sweeping process (3.11) we compute that

$$
D=(-1,1), \quad R=1, \quad U=\{0\}, \quad V=\mathbb{R}, \quad h(t)=0, \quad g(t)=1 \cdot(-1,1)(0,1)^{T} l(t)=l(t),
$$

where we used that the matrix $L$ satisfying (3.2) can be taken as $L=(0,1)^{T}$. Therefore,

$$
\Pi(t) \cap V=\left((1 / a)\left[c^{-}, c^{+}\right]-g(t)\right) \cap \mathbb{R}=(1 / a)\left[c^{-}, c^{+}\right]-l(t)
$$

and sweeping process (3.11) corresponding to elastoplastic system of Figure 5 takes the form

$$
-\dot{y} \in N_{\left[\frac{c^{-}}{a}-l(t), \frac{c^{+}}{a}-l(t)\right]}(y), \quad \text { where } \quad y(t)=e(t)-l(t),
$$

and $e(t)$ denotes the elastic elongation of the spring of Figure 5.

For the rest of the paper we work with an example where $\operatorname{dim} U>0$ (and we are particularly interested in the situation where $\operatorname{dim} U=1$ as it will satisfy the condition of our main result Thm. 5.4).

Example. Consider a one-dimensional network of 3 springs on 4 nodes with the kinematic matrix $D$ provided by the map

$$
D \xi=\left(\begin{array}{l}
\xi_{2}-\xi_{1} \\
\xi_{3}-\xi_{2} \\
\xi_{4}-\xi_{3}
\end{array}\right)=\left(\begin{array}{cccc}
-1 & 1 & 0 & 0 \\
0 & -1 & 1 & 0 \\
0 & 0 & -1 & 1
\end{array}\right)\left(\begin{array}{l}
\xi_{1} \\
\xi_{2} \\
\xi_{3} \\
\xi_{4}
\end{array}\right)
$$




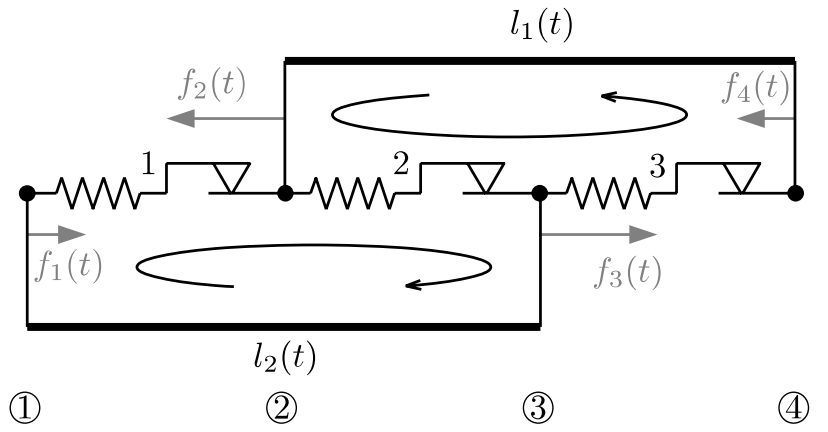

FIgURE 6. A one-dimensional network of 3 springs on 4 nodes with 2 length locking constraints. The circled figures stays for numbers of nodes. The regular figures are the numbers of springs. The figure shows just one possible option for the directions (and magnitudes) of the forces in gray color.

some $3 \times 3$ diagonal matrix $A$ of Hooke's coefficients and some intervals $\left[c_{j}^{-}, c_{j}^{+}\right], j \in \overline{1,3}$, of elasticity bounds. Assume that displacement-controlled loading $l(t) \in \mathbb{R}^{2}$ is given by the incidence vectors

$$
\left(R^{1}, R^{2}\right)=R=\left(\begin{array}{ll}
0 & 1 \\
1 & 1 \\
1 & 0
\end{array}\right),
$$

i.e. springs 2 and 3 connect the ends of $l_{1}(t)$ and springs 1 and 2 connect the ends of $l_{2}(t)$, see Figure 6 . To examine the shapes of the associated moving set $\Pi(t) \cap V$, we find out the eligible values of the function $h(t)$.

From (3.9) we conclude that eligible stress-controlled loading $f(t)$ leads to $h(t)$ given by

$$
h(t)=U_{\text {basis }} H(t)
$$

where $U_{\text {basis }}$ is the $m \times \operatorname{dim} U$-matrix of the vectors of a basis of $U$ and $H:[0, T] \rightarrow \mathbb{R}^{\operatorname{dim} U}$ is any absolutely continuous function. By (3.31) and (3.5), there should exist an $n \times(n-q-1)$-matrix $M$ such that

$$
R^{T} D M=0 \quad \text { and } \quad \operatorname{rank}(D M)=n-q-1
$$

which allows to introduce $U_{\text {basis }}$ as

$$
U_{\text {basis }}=D M \text {. }
$$

Getting back to the matrices $D$ and $R$ given by (3.32) and (3.33) one has $\operatorname{dim} U=n-q-1=4-2-1=1$, which means that even though the stress-controlled loading is comprised by four forces (one per node of the system from Fig. 6), only a scalar 1-dimensional parameter of these forces matters. A possible $4 \times 1$-matrix that solves (3.35), the respective $U_{\text {basis }}$ found from (3.36), and the respective function $h(t)$ given by (3.34) are then read as

$$
M=\left(\begin{array}{l}
0 \\
1 \\
0 \\
1
\end{array}\right), \quad U_{\text {basis }}=\left(\begin{array}{c}
1 \\
-1 \\
1
\end{array}\right), \quad h(t)=\left(\begin{array}{c}
1 \\
-1 \\
1
\end{array}\right) H(t)
$$




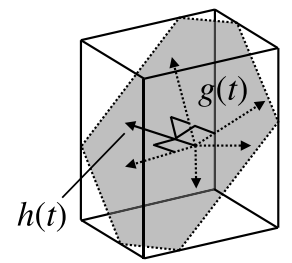

(a)

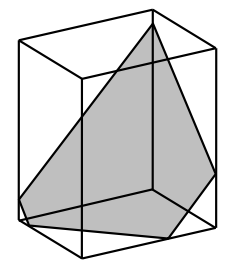

(b)

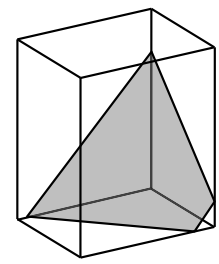

(c)

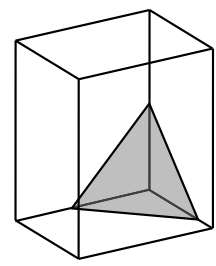

(d)

FIGURE 7. Shapes of the moving constraint $\Pi(t) \cap V$ for the sweeping process of the network of Figure 6 with parameters $c_{1}^{+}=-c_{1}^{-}=1, c_{2}^{+}=-c_{2}^{-}=1.3, c_{3}^{+}=-c_{3}^{-}=1.6, a_{1}=a_{2}=a_{3}=1$ for different values of stress-controlled loading $h(t)=(1,-1,1)^{T} t$ : a) $t=0$, b) $t=0.32$, c) $t=$ 0.5 , d) $t=0.8$. (a) Also features the possible directions of the function $g(t)$ (dotted vectors) and the possible direction of the function $h(t)$ (solid vector) that represent displacement-controlled and stress-controlled loading respectively.

where $H$ is an arbitrary absolutely continuous function from $[0, T]$ to $\mathbb{R}$. Figure 7 illustrates the shapes of $\Pi(t) \cap V$ for different constant values of $H(t)$, where according to (3.5) we considered

$$
V=\operatorname{Ker}\left(U_{b a s i s}^{T} A\right)=\left(\begin{array}{c}
a_{1} \\
-a_{2} \\
a_{3}
\end{array}\right)^{\perp} .
$$

\subsection{Bounds on the stress-controlled loading to satisfy the safe load condition}

Computational algorithms to verify safe load condition (3.15) for particular systems is a standard topic of computational geometry, see e.g. Bremner et al. [7]. In this section we derive analytic conditions which allow to spot classes of elastoplastic systems for which the safe load condition holds.

Proposition 3.9. In order for the safe load condition (3.15) of Theorem 3.1 to hold for some $t \in[0, T]$, it is sufficient to assume that

$$
-A h(t) \in C
$$

Proof. In order to show that (3.39) implies (3.15), it is sufficient to observe that $0 \in U^{\perp}$ and that (3.39) yields $0 \in C+A h(t)$.

Definition 3.10. We will say that a spring $i$ is blocked by displacement-controlled loadings, if the family of displacement-controlled loadings $\left\{l_{j}\right\}_{j=1}^{q}$ contains a chain that connects one end of spring $i$ with its other end.

For example, in the elastoplastic system of Figure 8, the chain of displacement-controlled loadings $l_{1}(t)$ and $l_{2}(t)$ connects the right end of spring 3 with its left end. Spring 3 is, therefore, blocked by displacement-controlled loadings in the sense of Definition 3.10.

Lemma 3.11. Assume that in a given elastoplastic system the number $q$ of displacement-controlled loadings is 2 less the number of nodes. If none of the springs of the elastoplastic system (2.1)-(2.5) is blocked by displacementcontrolled loadings, then

$$
x_{j} \neq 0 \text { for any } j \in \overline{1, m}, x \in U \backslash\{0\} .
$$




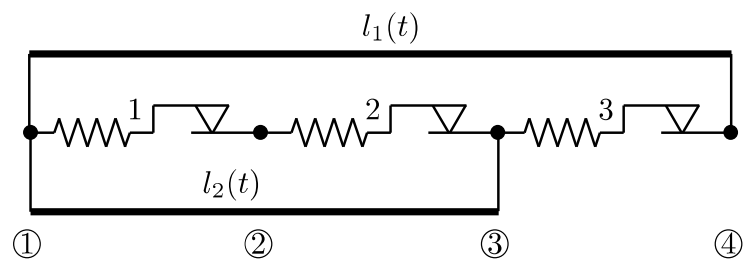

FiguRE 8. Illustration of a spring (spring 3 ) blocked by displacement-controlled loadings $\left(l_{1}(t)\right.$ and $\left.l_{2}(t)\right)$.

Proof. Recall, that $\xi_{\Phi_{k}}$ and $\xi_{\Psi_{k}}$ are the left and right endpoints respectively of the displacement-controlled constraint $l_{k}(t)$. Consider the matrix $D_{1}$ obtained from matrix $D$ by combining the column $\Phi_{k}$ and the column $\Psi_{k}$ as follows: 1) add the values of column $\Psi_{k}$ to the respective values of column $\Phi_{k}, 2$ ) delete the column $\Psi_{k}$. Then,

$$
\left\{D_{1} \xi: \xi \in \mathbb{R}^{n-1}\right\}=\left\{D \xi:\left(R^{k}\right)^{T} D \xi=0, \xi \in \mathbb{R}^{n}\right\} .
$$

Moreover, each row of $D$ has exactly one element 1 and -1 and none of the springs are blocked therefore at least one of each pair of summands at step 1) is zero. Matrix $D_{1}$ is the kinematic matrix for a new elastoplastic system that is obtained from elastoplastic system (2.1)-(2.5) by merging the nodes $\Phi_{k}$ and $\Psi_{k}$ together and, thus, by reducing the number of nodes by 1 . Accordingly, the new elastoplastic system features only $q-1$ displacement-controlled loadings and the indices $\left\{\Phi_{k}, \Psi_{k}\right\}_{k=1}^{q-1}$ are now from $\overline{1, n-1}$.

Repeating this process through all the incidence vectors $\left\{R^{k}\right\}_{k=1}^{q}$, where $q=n-2$ by Lemma 3.8, we obtain

$$
U=\left\{D \xi: R^{T} D \xi=0, \xi \in \mathbb{R}^{n}\right\}=\left\{\bar{D} \xi: \xi \in \mathbb{R}^{n-q}\right\}=\left\{\bar{D} \xi: \xi \in \mathbb{R}^{2}\right\},
$$

where $\bar{D}$ is the kinematic matrix of the reduced elastoplastic system that is obtained from the original one by merging node $\Phi_{k}$ with node $\Psi_{k}$ trough $k \in \overline{1, q}$.

Since the reduced elastoplastic system has only two nodes $(q=n-2)$, all the displacement-controlled constraints of the original system split into at most two connected components, which shrink into these two nodes under the proposed reduction process. If spring $j$ is not blocked by displacement-controlled loadings, then the endpoints of spring $j$ belong to different connected components introduced. Therefore, the endpoints of spring $j$ are two different nodes of the reduced elastoplastic system, which implies

$$
u_{j} \neq 0, j \in \overline{1, m}, \quad \text { for any } u=\bar{D} \xi \text { such that } \xi \in \mathbb{R}^{2}, \xi_{1} \neq \xi_{2} .
$$

If $\xi_{1}=\xi_{2}$ then $u=\bar{D} \xi=0$. The proof of the lemma is complete.

Proposition 3.12. Assume that the conditions of Lemma 3.11 hold. Let $\bar{u}$ be an arbitrary nonzero fixed vector of $U(\operatorname{dim} U=1$ by Lem. 3.8) and consider

$$
\bar{c}^{+}=\left(\begin{array}{c}
c_{1}^{\operatorname{sign}\left(\bar{u}_{1}\right)} \\
\vdots \\
c_{m}^{\operatorname{sign}\left(\bar{u}_{m}\right)}
\end{array}\right), \quad \bar{c}^{-}=\left(\begin{array}{c}
c_{1}^{-\operatorname{sign}\left(\bar{u}_{1}\right)} \\
\vdots \\
c_{m}^{-\operatorname{sign}\left(\bar{u}_{m}\right)}
\end{array}\right),
$$

where $c_{j}^{-1}$ denotes $c_{j}^{-}$and $c_{j}^{+1}$ denotes $c_{j}^{+}$. Then, for each fixed $t \geqslant 0$, the safe load condition (3.15) holds if and only if

$$
\left\langle\bar{u}, \bar{c}^{+}+A h(t)\right\rangle \cdot\left\langle\bar{u}, \bar{c}^{-}+A h(t)\right\rangle \leqslant 0
$$


Proof. We first show that (3.40) implies (3.15). Assume that (3.15) doesn't hold for some $t \in[0, T]$. Therefore, by convexity of $C$, either $\langle\bar{u}, x+A h(t)\rangle>0$ for all $x \in C$ or $\langle\bar{u}, x+A h(t)\rangle<0$ for all $x \in C$. In either case we conclude $\left\langle\bar{u}, \bar{c}^{+}+A h(t)\right\rangle \cdot\left\langle\bar{u}, \bar{c}^{-}+A h(t)\right\rangle>0$ because $\bar{c}^{+}, \bar{c}^{-} \in C$, which contradicts (3.40).

Let us now show that (3.15) implies (3.40). Indeed, since

$$
\bar{u}_{j} \bar{c}_{j}^{-} \leqslant \bar{u}_{j} c_{j}^{\alpha} \leqslant \bar{u}_{j} \bar{c}_{j}^{+}, \quad \text { for any } j \in \overline{1, m}, \alpha \in\{-1,+1\},
$$

we have

$$
\left\langle\bar{u}, \bar{c}^{-}+A h(t)\right\rangle \leqslant\langle\bar{u}, x+A h(t)\rangle \leqslant\left\langle\bar{u}, \bar{c}^{+}+A h(t)\right\rangle, \quad \text { for any } x \in C .
$$

The latter inequality takes the required form (3.40) when one plugs $x$ satisfying $\langle\bar{u}, x+A h(t)\rangle=0$, which exists because of (3.15).

Remark 3.13. Considering the left-hand-side of (3.40) as a polynomial $P(\langle\bar{u}, A h(t)\rangle)$ in $\langle\bar{u}, A h(t)\rangle$, we see that the branches of the polynomial are pointing upwards. Therefore, condition (3.40) is the requirement for $\langle\bar{u}, A h(t)\rangle$ to stay strictly between the roots of the polynomial. The roots of $P(\langle\bar{u}, A h(t)\rangle)$ are given by $\langle\bar{u}, A h(t)\rangle=$ $-\left\langle\bar{u}, \bar{c}^{-}\right\rangle$and $\langle\bar{u}, A h(t)\rangle=-\left\langle\bar{u}, \bar{c}^{+}\right\rangle$, where $\left\langle\bar{u}, \bar{c}^{-}\right\rangle \leqslant\left\langle\bar{u}, \bar{c}^{+}\right\rangle$by (3.41). Therefore, (3.40) is equivalent to

$$
-\left\langle\bar{u}, \bar{c}^{+}\right\rangle \leqslant\langle\bar{u}, A h(t)\rangle \leqslant-\left\langle\bar{u}, \bar{c}^{-}\right\rangle
$$

which highlights that (3.40) is a restriction on the magnitude of $\langle\bar{u}, A h(t)\rangle$.

Proposition 3.12 can be e.g. applied to the one-dimensional network of Figure 6 , where $\operatorname{dim} U=1$ as we noticed earlier.

Example (continued). For the elastoplastic system of Figure 6 one can consider $\bar{u}=(1,-1,1)^{T}$ and using (3.37) obtain

$$
\bar{c}^{+}=\left(\begin{array}{c}
c_{1}^{+} \\
c_{2}^{-} \\
c_{3}^{+}
\end{array}\right), \quad \bar{c}^{-}=\left(\begin{array}{c}
c_{1}^{-} \\
c_{2}^{+} \\
c_{3}^{-}
\end{array}\right), \quad\langle\bar{u}, A h(t)\rangle=\left(a_{1}+a_{2}+a_{3}\right) H(t)
$$

Based on Remark 3.13 the necessary and sufficient condition for safe load condition (3.40) to hold is then

$$
-c_{1}^{+}+c_{2}^{-}-c_{3}^{+} \leqslant\left(a_{1}+a_{2}+a_{3}\right) H(t) \leqslant-c_{1}^{-}+c_{2}^{+}-c_{3}^{-} .
$$

\subsection{Condition on the displacement-controlled loading to eliminate constant solutions}

Next proposition gives conditions to ensure that any point $x$ which belongs to the moving set $\Pi(t) \cap V$ of sweeping process (3.11) at some time $t=t_{1}$ will lie outside $\Pi(t) \cap V$ at time $t=t_{2}$. These conditions will, therefore, rule out the existence of constant solutions.

Proposition 3.14. Assume that conditions of Theorem 3.1 hold. If

$$
\left\|A^{-1} c^{-}-A^{-1} c^{+}\right\|_{A}<\left\|g\left(t_{1}\right)-g\left(t_{2}\right)\right\|_{A},
$$

for some $0 \leqslant t_{1}<t_{2} \leqslant T$, where

$$
\|x\|_{A}=\sqrt{\langle x, A x\rangle}, \quad c^{-}=\left(c_{1}^{-}, \ldots, c_{m}^{-}\right)^{T}, \quad c^{+}=\left(c_{1}^{+}, \ldots, c_{m}^{+}\right)^{T},
$$


then sweeping process (3.11) doesn't have any solutions that are constant on $\left[t_{1}, t_{2}\right]$. In other words, any solution $y$ of (3.11) on $\left[t_{1}, t_{2}\right]$ with the initial condition $y\left(t_{1}\right) \in \Pi(t) \cap V$ sticks to the boundary of $\Pi(t) \cap V$ and stays sliding along this boundary beginning some time $t_{*} \in\left[t_{1}, t_{2}\right)$.

Proof. The claim follows by showing that

$$
\left(\Pi\left(t_{1}\right) \cap V\right) \cap\left(\Pi\left(t_{2}\right) \cap V\right)=\emptyset .
$$

Since $h(t) \in U$ we have

$$
\Pi(t) \cap V=\left(A^{-1} C+h(t)-g(t)\right) \cap V \subset P_{V} A^{-1} C-g(t), \quad t \in\left[t_{1}, t_{2}\right],
$$

and it is sufficient to prove that the sets

$$
P_{V} A^{-1} C-g\left(t_{1}\right) \text { and } P_{V} A^{-1} C-g\left(t_{2}\right) \text { don't intersect. }
$$

The latter will hold, if the diameter of the set $P_{V} A^{-1} C$ is smaller than the distance between $g\left(t_{1}\right)$ and $g\left(t_{2}\right)$, which fact will now be established.

Since $P_{V}$ is the orthogonal projection in the sense of the scalar product $(x, y)_{A}=\langle x, A y\rangle$, we have (see e.g. Conway [17], Thm. 2.7 b)

$$
\left\|P_{V} x\right\|_{A} \leqslant\|x\|_{A}, \quad x \in \mathbb{R}^{m} .
$$

Fix $c_{1}, c_{2} \in C$ and denote by $c_{1, j}, c_{2, j}, j \in \overline{1, m}$, the components of these vectors. By the definition of $C$, we have

$$
\left|c_{1, j}-c_{2, j}\right| \leqslant\left|c_{j}^{-}-c_{j}^{+}\right|, \quad j \in \overline{1, m}
$$

Therefore, for any $c_{1}, c_{2} \in C$,

$$
\begin{gathered}
\left\|P_{V}\left(A^{-1} c_{1}-A^{-1} c_{2}\right)\right\|_{A} \leqslant\left\|A^{-1} c_{1}-A^{-1} c_{2}\right\|_{A}=\sum_{j=1}^{m} \frac{1}{a_{j}}\left|c_{1, j}-c_{2, j}\right|^{2} \\
\leqslant \sum_{j=1}^{m} \frac{1}{a_{j}}\left|c_{j}^{-}-c_{j}^{+}\right|^{2}=\left\|A^{-1} c^{-}-A^{-1} c^{+}\right\|_{A}
\end{gathered}
$$

The proof of the proposition is complete.

Remark 3.15. Note, the left-hand-side in the squared inequality (3.44) from the statement of Proposition 3.14 can be computed as

$$
\left\|A^{-1} c^{-}-A^{-1} c^{+}\right\|_{A}^{2}=\left\langle c^{-}-c^{+}, A^{-1}\left(c^{-}-c^{+}\right)\right\rangle .
$$

In what follows we show which kind of computations is required to verify the condition of Proposition 3.14 in practice.

Example (continued). Given the elastoplastic system of Figure 6, our goal is to compute the effective displacement-controlled loading $g(t)$ of (3.8).

For the term $P_{V} D L$ of (3.8) observe, that there exists a $\operatorname{dim} V \times q$-matrix $\bar{L}$ such that

$$
V_{\text {basis }} \bar{L}=P_{V} D L
$$


where $V_{\text {basis }}$ is the $m \times \operatorname{dim} V$-matrix of the vectors of a basis of $V$. The $i$ th column of matrix $\bar{L}$ is the vector of the coordinates of the respective vector $P_{V} D L^{i} \in V$ in the basis $V_{\text {basis }}$, where $L^{i}$ stays for the $i$ th column on matrix $L$. Formula (3.8) can therefore be rewritten as

$$
g(t)=V_{b a s i s} \bar{L} l(t)
$$

Computing the effective displacement-controlled loading $g(t)$ has hereby been turned into computing $V_{\text {basis }}$ and $\bar{L}$.

By (3.31),

$$
\operatorname{dim} V=m-n+q+1
$$

and according to (3.38), $V_{\text {basis }}$ is an arbitrary matrix of $\operatorname{dim} V$ linearly independent columns that solves

$$
\left(U_{\text {basis }}\right)^{T} A V_{\text {basis }}=0
$$

For the particular matrices (3.32), using the earlier computed $U_{\text {basis }}$, see (3.37), one gets $\operatorname{dim} V=2,\left(U_{\text {basis }}\right)^{T}=$ $(1-11)$, and a possible solution to $(3.48)$ is

$$
V_{\text {basis }}=\left(\begin{array}{cc}
1 / a_{1} & 0 \\
1 / a_{2} & 1 / a_{2} \\
0 & 1 / a_{3}
\end{array}\right)
$$

To find $\bar{L}$, we observe that by $(3.2)$, for any $\xi \in \mathbb{R}^{q}$, we have

$$
P_{V} D L \xi=D L \xi-P_{U} D L \xi \in D \mathbb{R}^{n}
$$

as $P_{U} D L \xi \in D \mathbb{R}^{n}$ by definition of $U$. Combining this relation with (3.2) and (3.45) one gets the following equations for $\bar{L}$ :

$$
\begin{aligned}
R^{T} V_{\text {basis }} \bar{L} & =I_{q \times q}, \\
V_{\text {basis }} \bar{L} \mathbb{R}^{q} & \subset D \mathbb{R}^{n},
\end{aligned}
$$

from which $\bar{L}$ can be found. In Appendix D we offer a diagram (Fig. D.1) showing the construction of $V_{\text {basis }} \bar{L}$ graphically.

For a specific matrix $D$ given by (3.32), one has $D \mathbb{R}^{4}=\mathbb{R}^{3}$ (i.e. there is no geometric constraint coming from the graph of springs in this case) and so (3.50) holds for any matrix $\bar{L}$. The matrix $\bar{L}$ is therefore a $2 \times 2-$ matrix that solves (3.49), which has a unique solution

$$
\bar{L}=\left(\begin{array}{cc}
1 / a_{2} & 1 / a_{2}+1 / a_{3} \\
1 / a_{1}+1 / a_{2} & 1 / a_{2}
\end{array}\right)^{-1}
$$

Formula (3.46), in particular, implies that, for the network of springs of Figure 6 (where $\operatorname{dim} V=q=2$ ), the displacement-controlled constraints are capable to execute any desired motion of $C(t)$ in $V$.

Applying Proposition 3.14 and Remark 3.15, we obtain the following condition for non-existence of constant solutions. The elastoplastic system of Figure 6 cannot have constant solutions on $[0, T]$, if there exist $t_{1}, t_{2} \in[0, T]$ 
such that

$$
\sum_{j=1}^{3} \frac{1}{a_{j}}\left(c_{j}^{+}-c_{j}^{-}\right)^{2}<\left\|V_{\text {basis }} \bar{L}\left(l\left(t_{1}\right)-l\left(t_{2}\right)\right)\right\|_{A}^{2} .
$$

\section{Convergence to a Periodic attractor}

\subsection{Convergence in the case of a moving constraint given by an intersection of translationally moving convex sets}

In this section we establish convergence properties of a general sweeping process

$$
-\dot{x} \in N_{C(t)}^{0}(x), \quad x \in E,
$$

where $E$ is a $d$-dimensional linear vector space, $C(t) \subset E$ is convex closed set for any $t$, and

$$
N_{C}^{0}(x)= \begin{cases}\left\{\xi \in E:(\xi, c-x)_{0} \leqslant 0, \text { for any } c \in C\right\}, & \text { if } x \in C, \\ \emptyset, & \text { if } x \notin C,\end{cases}
$$

where $(\cdot, \cdot)_{0}$ is some inner product in $E$. These convergence properties are then refined in Section 4.3 in the context of the particular sweeping process (3.11).

A set-valued function $t \mapsto C(t), t \in[0, \infty)$, is called globally Lipschitz continuous, if

$$
d_{H}\left(C\left(t_{1}\right), C\left(t_{2}\right)\right) \leqslant L_{C}\left|t_{1}-t_{2}\right|, \text { for all } t_{1}, t_{2} \geq 0 \text {, and for some } L_{C}>0,
$$

where $d_{H}\left(C_{1}, C_{2}\right)$ is the Hausdorff distance between two closed sets $C_{1}, C_{2} \in E$ defined as

$$
d_{H}\left(C_{1}, C_{2}\right)=\max \left\{\sup _{x \in C_{2}} \operatorname{dist}\left(x, C_{1}\right), \sup _{x \in C_{1}} \operatorname{dist}\left(x, C_{2}\right)\right\}
$$

with $\operatorname{dist}(x, C)=\inf \{|x-c|: c \in C\}$.

Recall, if $C(t)$ is a globally Lipschitz continuous function with nonempty closed convex values from $E$, then the solution $x(t)$ of sweeping process (4.1) with any initial condition $x\left(t_{0}\right)=x_{0}$ is uniquely defined on $\left[t_{0}, \infty\right)$ in the sense that $x(t)$ is a Lipschitz continuous function that verifies (4.1) for a.a. $t \in\left[t_{0}, \infty\right.$ ) (see e.g. Kunze and Monteiro Marques [38]).

Let us use $t \mapsto X\left(t, x_{0}\right)$ to denote the solution of sweeping process (4.1) that takes the value $x_{0}$ at time 0 . In what follows, we consider the set of $T$-periodic solutions of (4.1)

$$
\begin{aligned}
X & =\left\{X\left(\cdot, x_{0}\right): x_{0}=X\left(T, x_{0}\right)\right\} \subset C([0, \infty), E) \\
X(t) & =\left\{X\left(t, x_{0}\right): x_{0}=X\left(T, x_{0}\right)\right\} \subset E
\end{aligned}
$$

and prove that, for $T$-periodic moving constraint $C(t)$, the set $X(t)$ attracts all the solutions of (4.1).

Remark 4.1. Note, if $t \mapsto C(t)$ is $T$-periodic, then it holds $X(T, C(0)) \subset C(0)$ and so the continuous map $x_{0} \mapsto X\left(T, x_{0}\right)$ has at least one fixed point in $C(0)$ by the Brouwer fixed point theorem (see Thm. 3.1 in [34]). In particular, if $t \mapsto C(t)$ is $T$-periodic, then $X \neq \emptyset$.

Definition 4.2. A set-valued function $t \mapsto Y(t)$ is a global attractor of sweeping process (4.1), if $\operatorname{dist}(x(t), Y(t)) \rightarrow 0$ as $t \rightarrow \infty$ for any solution $x$ of sweeping process (4.1).

Finally, we denote by $\operatorname{ri}(C)$ the relative interior of a convex set $C \subset E$, see Rockafellar ([50], Sect. 6). 
Theorem 4.3. Let $t \mapsto C(t)$ be a Lipschitz continuous uniformly bounded T-periodic set-valued function with nonempty closed convex values from $E$. Let $t \mapsto X(t)$ be the set of T-periodic solutions of sweeping process (4.1) as defined in (4.6). Then, $X(t)$ is closed and convex for all $t \in[0, T]$. If, in addition, $C(t)$ is an intersection of closed convex sets $C_{j}$ (some of them, say, first p sets, may be polyhedral) that undergo just translational motions

$$
C(t)=\bigcap_{j=1}^{M}\left(C_{j}+c_{j}(t)\right)
$$

where $c_{j}(t)$ are single-valued T-periodic Lipschitz functions such that

$$
\bigcap_{j=1}^{p}\left(C_{j}+c_{j}(t)\right) \cap \bigcap_{j=p+1}^{M}\left(\operatorname{ri}\left(C_{j}\right)+c_{j}(t)\right) \neq \emptyset, \quad t \in[0, T],
$$

then

$$
\dot{x}(t)=\dot{y}(t), \quad \text { for any } x, y \in X \text { and for a.a. } t \in[0, T]
$$

and $X(t)$ is a global attractor of (4.1).

The theorem, in particular, implies that $X(t)$ cannot contain non-constant solutions, if it contains at least one constant solution.

The proof of theorem 4.3 is split into 3 lemmas. Lemma 4.4 establishes the convexity of $X$ (closedness of $X(t)$ follows from the continuous dependence of solutions of (4.1) on the initial condition, see Corollary 1 in [38]). Lemma 4.5 proves the statement (4.9). Finally, the global attractivity of $X(t)$ is given by Theorem 4.6 which is an extension of a result from Krejci [36] for convex sets (4.7).

In what follows, $\|\cdot\|_{0}$ is the norm induced by the scalar product in $E$, i.e.

$$
\|x\|_{0}=\sqrt{(x, x)_{0}}
$$

Lemma 4.4. Let $t \mapsto C(t)$ be a Lipschitz continuous set-valued function with nonempty closed convex values from $E$. Then, both $X(t) \subset E$ and $X \subset C([0, \infty), E)$ are convex. In addition, for any $x, y \in X \subset C([0, \infty), E)$

$$
\|x(t)-y(t)\|_{0} \text { is constant in } t .
$$

Proof. Let $x, y \in X$. Due to monotonicity of $N_{C(t)}^{0}(x)$ in $x$ for each $t \geqslant 0$, the distance $t \mapsto\|x(t)-y(t)\|_{0}$ cannot increase (see e.g. [38], Cor. 1). Notice, that $t \mapsto\|x(t)-y(t)\|_{0}$ cannot decrease, otherwise it cannot be periodic, so (4.11) follows.

For any $\theta \in(0,1)$ the initial condition $\theta x(0)+(1-\theta) y(0)$ belongs $C(0)$ by convexity of $C$. Let $x_{\theta}$ be the corresponding solution. Since $t \mapsto\left\|x(t)-x_{\theta}(t)\right\|_{0}$ and $t \mapsto\left\|x_{\theta}(t)-y(t)\right\|_{0}$ are also non-increasing, then

$$
\left\|x(t)-x_{\theta}(t)\right\|_{0}+\left\|y(t)-x_{\theta}(t)\right\|_{0} \leqslant\left\|x(0)-x_{\theta}(0)\right\|_{0}+\left\|y(0)-x_{\theta}(0)\right\|_{0}=\|x(0)-y(0)\|_{0}=\|x(t)-y(t)\|_{0} .
$$

On the other hand, the triangle inequality yields

$$
\left\|x(t)-x_{\theta}(t)\right\|_{0}+\left\|y(t)-x_{\theta}(t)\right\|_{0} \geqslant\|x(t)-y(t)\|_{0}
$$


and we have

$$
\left\|x(t)-x_{\theta}(t)\right\|_{0}+\left\|x_{\theta}(t)-y(t)\right\|_{0}=\|x(t)-y(t)\|_{0} \equiv \text { const. }
$$

Because none of the terms $\left\|x(t)-x_{\theta}(t)\right\|_{0},\left\|x_{\theta}(t)-y(t)\right\|_{0}$ can increase, both of them remain constant and positive (due to the choice of $x_{\theta}(0)$ ). Moreover, by strict convexity of the inner product space (Narici-Beckenstein [46], Thms. 16.1.4(d) and 16.1.5, see also Appendix B) there exists $\gamma(t)>0$ such that

$$
x_{\theta}(t)-y(t)=\gamma(t)\left(x(t)-x_{\theta}(t)\right) .
$$

We solve for $x_{\theta}$ :

$$
x_{\theta}(t)=\frac{\gamma(t)}{1+\gamma(t)} x(t)+\frac{1}{1+\gamma(t)} y(t)
$$

and substitute it to the second difference in (4.12):

$$
\left\|x(t)-x_{\theta}(t)\right\|_{0}=\frac{1}{1+\gamma(t)}\|x(t)-y(t)\|_{0}
$$

Both distances $\left\|x(t)-x_{\theta}(t)\right\|_{0}$ and $\|x(t)-y(t)\|_{0}$ are constant, hence $\gamma(t)$ is constant as well, which means that $\gamma(t)=\gamma(0)$ and due to the choice of $x_{\theta}(0)$ expression (4.13) becomes

$$
x_{\theta}(t)=\theta x(t)+(1-\theta) y(t), \quad \text { for any } \theta \in[0,1] .
$$

This formula, in particular, implies that $x_{\theta}$ is $T$-periodic. The proof of convexity of $X$ is complete.

Lemma 4.5. Let $t \mapsto C(t)$ be a set-valued function of the form (4.7)-(4.8) with convex closed $C_{j}$ and Lipschitzcontinuous single valued $c_{j}(t)$. Let $x$ and $y$ be two solutions of sweeping process (4.1) defined on $[0, T]$ such that (4.11) holds for them. Then for almost all $t \in[0, T]$

$$
\dot{x}(t)=\dot{y}(t) .
$$

Proof. The properties (4.7)-(4.8) imply ([50], Cor. 23.8.1, see also Appendix B) that

$$
N_{C(t)}^{0}(x)=\sum_{j=1}^{M} N_{C_{j}+c_{j}(t)}^{0}(x), \quad \text { for all } x \in C(t) \text { and for all } t \in[0, T] .
$$

Let $t \in(0, T)$ be such that $\dot{x}(t), \dot{y}(t), \dot{c}_{j}(t), j \in \overline{1, M}$, exist and (4.1) holds. Property (4.14) allows to spot $\dot{x}_{j}^{t}$, $\dot{y}_{j}^{t}, j \in \overline{1, M}$, such that

$$
\begin{gathered}
\dot{x}(t)=\sum_{j=1}^{M} \dot{x}_{j}^{t}, \quad-\dot{x}_{j}^{t} \in N_{C_{j}+c_{j}(t)}^{0}(x(t)), \quad j \in \overline{1, M}, \\
\dot{y}(t)=\sum_{j=1}^{M} \dot{y}_{j}^{t}, \quad-\dot{y}_{j}^{t} \in N_{C_{j}+c_{j}(t)}^{0}(y(t)), \quad j \in \overline{1, M} .
\end{gathered}
$$


To show that $\|\dot{x}(t)-\dot{y}(t)\|_{0}=0$, consider

$$
\begin{aligned}
\|\dot{x}(t)-\dot{y}(t)\|_{0}^{2}= & (\dot{x}(t)-\dot{y}(t), \dot{x}(t)-\dot{y}(t))_{0} \\
= & \sum_{j=1}^{M}\left(\dot{x}_{j}^{t}, \dot{x}(t)-\dot{c}_{j}(t)\right)_{0}+\sum_{j=1}^{M}\left(\dot{y}_{j}^{t}, \dot{y}(t)-\dot{c}_{j}(t)\right)_{0} \\
& -\sum_{j=1}^{M}\left(\dot{x}_{j}^{t}, \dot{y}(t)-\dot{c}_{j}(t)\right)_{0}-\sum_{j=1}^{M}\left(\dot{y}_{j}^{t}, \dot{x}(t)-\dot{c}_{j}(t)\right)_{0} .
\end{aligned}
$$

For the value of $t \in(0, T)$ as fixed above, we now prove that each of sums in (4.15)-(4.16) vanish.

Step 1. Vanishing sums in (4.15). Fix $j \in \overline{1, M}$. By the definition of normal cone,

$$
\left(\dot{x}_{j}^{t}, z+c_{j}(t)-x(t)\right)_{0} \geqslant 0 \text { and }\left(\dot{y}_{j}^{t}, z+c_{j}(t)-y(t)\right)_{0} \geqslant 0 \text { for all } z \in C_{j} .
$$

Considering $z=x(t+h)-c_{j}(t+h) \in C_{j}$, we observe that the function

$$
f(h)=\left(\dot{x}_{j}^{t}, x(t+h)-x(t)-\left(c_{j}(t+h)-c_{j}(t)\right)\right)_{0}
$$

is non-negative in a neighborhood of zero. Since $f(0)=0$, we conclude that $0=f^{\prime}(0)=\left(\dot{x}_{j}^{t}, \dot{x}(t)-\dot{c}_{j}(t)\right)_{0}$ (if $f^{\prime}(0) \neq 0$, then $f$ would take negative values in the neighborhood of 0 due to $\left.f(0)=0\right)$. The relation $\left(\dot{y}_{j}^{t}, \dot{y}(t)-\dot{c}_{j}(t)\right)_{0}=0$ can be proved by analogy using the second inequality of (4.17).

Step 2. Vanishing sums in (4.16). We claim that

$$
\sum_{j=1}^{M}\left(\dot{x}_{j}^{t}, z_{j}+c_{j}(t)-y(t)\right)_{0} \geqslant 0, \sum_{j=1}^{M}\left(\dot{y}_{j}^{t}, z_{j}+c_{j}(t)-x(t)\right)_{0} \geqslant 0, z_{j} \in C_{j},
$$

so that the arguments of Step 1 apply to

$$
f(h)=\sum_{j=1}^{M}\left(\dot{x}_{j}^{t}, y(t+h)-y(t)+c_{j}(t)-c_{j}(t+h)\right)_{0}
$$

(similarly for the second sum of (4.18) with $\left.z_{j}=x(t+h)-c_{j}(t+h)\right)$ to show that the sums in (4.16) vanish. To establish (4.18), we first rewrite it as

$$
\sum_{j=1}^{M}\left(\dot{x}_{j}^{t}, z_{j}+c_{j}(t)\right)_{0}-(\dot{x}(t), y(t))_{0} \geqslant 0, \quad \sum_{j=1}^{M}\left(\dot{y}_{j}^{t}, z_{j}+c_{j}(t)\right)_{0}-(\dot{y}(t), x(t))_{0} \geqslant 0
$$

and then prove that

$$
(\dot{x}(t), y(t))_{0}=(\dot{x}(t), x(t))_{0} \quad \text { and } \quad(\dot{y}(t), x(t))_{0}=(\dot{y}(t), y(t))_{0}, \quad t \in[0, T]
$$

so that (4.18) becomes a consequence of (4.17). To prove (4.19) we use (4.11) and observe that

$$
0=\frac{\mathrm{d}}{\mathrm{d} t}\|x(t)-y(t)\|_{0}^{2}=-(\dot{x}(t), y(t)-x(t))_{0}-(\dot{y}(t), x(t)-y(t))_{0} .
$$


But $x(t), y(t) \in C(t)$ and both these functions are solutions of sweeping process (4.1). Therefore, $(\dot{x}(t), y(t)-x(t))_{0} \geqslant 0$ and $(\dot{y}(t), x(t)-y(t))_{0} \geqslant 0$, which implies (4.19).

The proof of the lemma is complete.

We acknowledge that the idea of the proof of Step 1 of Lemma 4.5 has been earlier used by Krejci in the proof of Theorem 3.14 in [36], which would suffice for the proof when $M=1$. The achievement of Lemma 4.5 is in considering $M>1$, thus the new Step 2. Accordingly, the proof of the next theorem follows the lines of Theorem 3.14 in [36] with Lemma 4.5 used to justify (C.6), which is the place of the proof that needed further arguments when moving to $M>1$. We present a proof for completeness (Appendix C) also because [36] employs slightly different notations. The theorem effectively states that any bounded solution of a $T$-periodic sweeping process is asymptotically $T$-periodic, which facts is known in differential equations as Massera's theorem [44].

Theorem 4.6. (Massera-Krejci theorem for sweeping processes with a moving set of the form $\left.\boldsymbol{C}(\boldsymbol{t})=\cap_{j=1}^{M}\left(C_{j}+\boldsymbol{c}_{\boldsymbol{j}}(\boldsymbol{t})\right)\right)$ Let $t \mapsto C(t)$ be a set-valued uniformly bounded function of the form (4.7)-(4.8) with convex closed $C_{j}$ and Lipschitz-continuous single-valued T-periodic $c_{j}(t)$. Then the set $X(t)$ of T-periodic solutions of (4.1) is a global attractor of (4.1).

The proof of Theorem 4.6 is given in Appendix C.

Lemma 4.5 implies that each set $X(t), t \in[0, T]$, is just a displacement of the set $X(0)$ by a vector. Specifically, the following statement holds.

Corollary 4.7. Let $t \mapsto C(t)$ be a Lipschitz continuous uniformly bounded T-periodic set-valued function with nonempty closed convex values from $E$. Let $t \mapsto X(t)$ be the set of $T$-periodic solutions of sweeping process (4.1) as defined in (4.6). Then, there exists a Lipschitz continuous T-periodic function $\bar{x}:[0, \infty) \rightarrow E$ such that

$$
x(t)=x(0)+\bar{x}(t), \quad t \in[0, T], x \in X .
$$

In particular,

$$
X(t)=X(0)+\bar{x}(t), \quad t \in[0, T]
$$

and

$$
x(t) \in \operatorname{ri}(X(t)) \quad \text { if and only if } \quad x(0) \in \operatorname{ri}(X(0)) .
$$

Proof. Since any Lipschitz continuous function $x(t)$ can be represented as $x(t)=x(0)+\int_{0}^{t} \dot{x}(t) d t$, the existence of $\bar{x}$ verifying the first two assertions of the corollary follows directly from Lemma 4.5. The assertion about the relative interior follows by observing that, for any $v \in E, x(t) \in \operatorname{ri}(X(t))$ if and only if $x(t)+v \in \operatorname{ri}(X(t)+v)$, so one can take $v=-\bar{x}(t)$.

An interested reader can note that sweeping process (4.1) with $M=1$ converts to a perturbed sweeping process $-\dot{\xi} \in N_{C_{1}}^{0}(\xi)+\dot{c}_{1}(t)$ with an immovable constraint by the change of the variables $\xi(t)=y(t)-c_{1}(t)$, while it is not clear whether or not (4.1) converts to a perturbed sweeping process with a constant constraint when $M>1$. This further highlights the difference between the cases $M=1$ and $M>1$ as long as potential alternative methods of analysis of the dynamics of (4.1) are concerned.

\subsection{Strengthening of the conclusion of Section 4.1 in the case of a moving constraint given by a polyhedron with translationally moving facets}

When applied to a one-dimensional network of elastoplastic springs (2.1)-(2.5), the existence of a periodic attractor $X(t)$ for the associated sweeping process (3.11) follows from Theorem 4.3. A new geometric property of $X(t)$ that comes with considering the sweeping process (3.11) is due to the polyhedral shape of the moving 

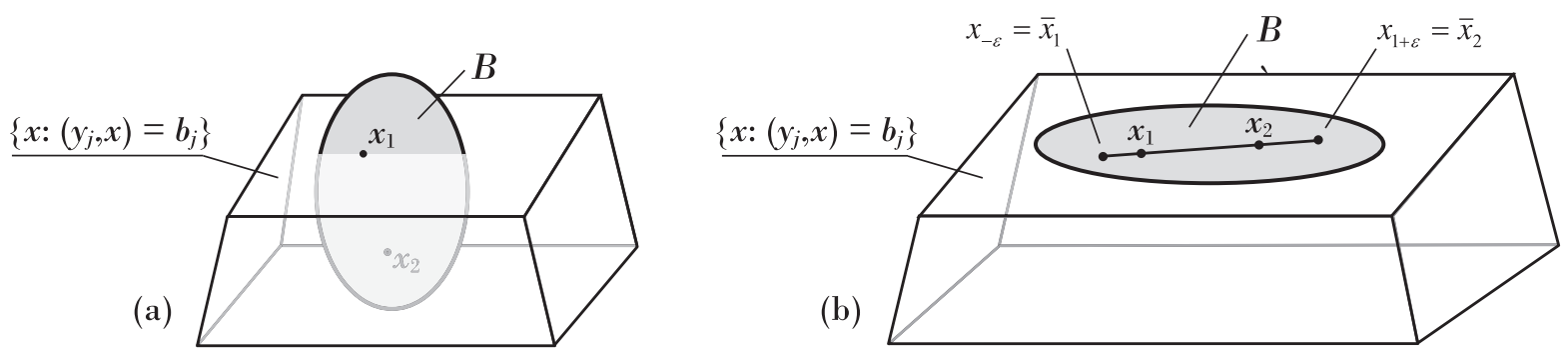

Figure 9. Illustration of the statement of Lemma 4.9. (a) An impossible configuration, which is ruled out by assumption (4.22). Assumption (4.22) forbids $B$ to stick out from the polyhedron; (b) The only possible configuration within the restriction given by $(4.22)$ and $\left(y_{j}, x_{1}\right)_{0}=b_{j}$. The polyhedron in both figures is $\bigcap_{j=1}^{M}\left\{x \in E:\left(y_{j}, x\right)_{0} \leqslant b_{j}\right\}$.

constraint $\Pi(t) \cap V$, see Section 3.2. Theorem 4.8 below states that even if $X(t)$ consists of several periodic solutions, they all exhibit certain identical behavior.

As earlier, let $E$ be a finite-dimensional linear vector space equipped with a scalar product $(\cdot, \cdot)_{0}$ and let

$$
\operatorname{ri}(X)=\{x \in X: x(0) \in \operatorname{ri}(X(0))\}
$$

which notations is justified by Corollary 4.7 .

Theorem 4.8. Assume that a uniformly bounded set-valued function $t \mapsto C(t)$ is given by

$$
C(t)=\bigcap_{j=1}^{m}\left\{x \in E: c_{j}^{-}(t) \leqslant\left(n_{j}, x\right)_{0} \leqslant c_{j}^{+}(t)\right\}, \quad t \geqslant 0,
$$

where $c_{j}^{-}, c_{j}^{+}$are single-valued T-periodic globally Lipschitz continuous functions, $n_{j}$ are given vectors from $E$. Then the set $X(t)$ of T-periodic solutions of sweeping process (4.1) is the global attractor of (4.1). Furthermore, $X \subset C([0, \infty), E)$ is closed and convex, and all the interior solutions of $X$ follow the same pattern of motion in the sense that

$$
J(t, x(t))=J(t, y(t)), \quad \text { for all } x, y \in \operatorname{ri}(X), t \geqslant 0,
$$

where $J(t, x)$ is the active set of the polyhedron $C(t)$ given by

$$
J(t, x)=\left\{j \in \overline{-m,-1}:\left(n_{-j}, x\right)_{0}=c_{-j}^{-}(t)\right\} \cup\left\{i \in \overline{1, m}:\left(n_{j}, x\right)_{0}=c_{j}^{+}(t)\right\} .
$$

Theorem 4.8 is a corollary of Theorem 4.3 except for the property (4.21) which comes from the polyhedral shape of the moving constraint $C(t)$. The property (4.21) follows from the following general result.

Lemma 4.9. Consider an arbitrary convex set B embedded into a convex polyhedron:

$$
B \subset \bigcap_{j=1}^{M}\left\{x \in E:\left(y_{j}, x\right)_{0} \leqslant b_{j}\right\}
$$

where $y_{j} \in E$ and $b_{j} \in \mathbb{R}$. If $x_{1}, x_{2} \in \operatorname{ri}(B)$, then, for all $j \in \overline{1, M}$,

$$
\left(y_{j}, x_{1}\right)_{0}=b_{j} \quad \text { if and only if } \quad\left(y_{j}, x_{2}\right)_{0}=b_{j} .
$$


Geometrically, Lemma 4.9 is saying that if $x_{1}$ belongs to a facet of the polyhedron $\bigcap_{j=1}^{M}\left\{x \in E:\left(y_{j}, x\right)_{0} \leqslant b_{j}\right\}$ and $B$ doesn't stick out from the polyhedron (i.e. Fig. 9a is not allowed), then $x_{2}$ belongs to the same facet as $x_{1}$ (as in Fig. 9b).

Proof. Assume that $\left(y_{j}, x_{1}\right)_{0}=b_{j}$ for some $x_{1} \in \operatorname{ri}(B)$ and some $j \in \overline{1, M}$, i.e. assume that the point $x_{1}$ belongs to the facet $\left\{x:\left(y_{j}, x\right)_{0}=b_{j}\right\}$. Let $x_{2}$ be any other point such that $x_{2} \in \operatorname{ri}(B)$ as Figure 9 a illustrates. We claim that if (4.22) holds ( $B$ doesn't stick out from the polyhedron, see Fig. 9 ), then $\left(y_{j}, x_{2}\right)_{0}=b_{j}$.

For any $\theta \in \mathbb{R}$, define $x_{\theta}$ as

$$
x_{\theta}=\theta x_{1}+(1-\theta) x_{2} .
$$

Since $x_{1}, x_{2} \in \operatorname{ri}(B)$, there exists $\varepsilon>0$ such that $x_{-\varepsilon} \in \operatorname{ri}(B)$ and $x_{1+\varepsilon} \in \operatorname{ri}(B)$, see Figure $9 \mathrm{~b}$. Put $\bar{x}_{1}=x_{-\varepsilon}$, $\bar{x}_{2}=x_{1+\varepsilon}$. Then there exist $\theta_{1}, \theta_{2} \in(0,1), \theta_{1} \neq \theta_{2}$, such that

$$
x_{1}=\theta_{1} \bar{x}_{1}+\left(1-\theta_{1}\right) \bar{x}_{2}, \quad x_{2}=\theta_{2} \bar{x}_{1}+\left(1-\theta_{2}\right) \bar{x}_{2} .
$$

Then, taking the scalar product of the first formula of (4.24) with $y_{j}$, replacing $b_{j}$ by $b_{j}=\theta_{1} b_{j}+\left(1-\theta_{1}\right) b_{j}$, and redistributing the terms, one gets

$$
\theta_{1}\left(\left(y_{j}, \bar{x}_{1}\right)_{0}-b_{j}\right)=-\left(1-\theta_{1}\right)\left(\left(y_{j}, \bar{x}_{2}\right)_{0}-b_{j}\right) .
$$

Since $\bar{x}_{1}, \bar{x}_{2} \in B$, one has $\left(y_{j}, \bar{x}_{1}\right)_{0}-b_{j} \leqslant 0$ and $\left(y_{j}, \bar{x}_{2}\right)_{0}-b_{j} \leqslant 0$. Therefore formula (4.25) can only hold when both $\left(y_{j}, \bar{x}_{1}\right)_{0}-b_{j}$ and $\left(y_{j}, \bar{x}_{2}\right)_{0}-b_{j}$ vanish. Hence

$$
\left(y_{j}, x_{2}\right)_{0}=\theta_{2}\left(y_{j}, \bar{x}_{1}\right)_{0}+\left(1-\theta_{2}\right)\left(y_{j}, \bar{x}_{2}\right)_{0}=\theta_{2} b_{j}+\left(1-\theta_{2}\right) b_{j}=b_{j} .
$$

The reverse implication in (4.23) can be proved by analogy.

\subsection{Application: an analytic condition for the convergence of the stresses of elastoplastic systems to an attractor}

Let $J_{C}(x)$ be the active set of the parallelepiped $C$, i.e.

$$
J_{C}(x)=\left\{j \in \overline{-m,-1}: x_{-j}=c_{-j}^{-}\right\} \cup\left\{j \in \overline{1, m}: x_{j}=c_{j}^{+}\right\} .
$$

A direct consequence of Theorem 4.8 is the following result about asymptotic behavior of the stresses of the elastoplastic system (2.1)-(2.5).

Theorem 4.10. Let the conditions of Theorem 3.1 hold and both displacement-controlled and stress-controlled loadings are T-periodic. Then, for any initial condition at $t=0$, the stress-vector $s(t)=\left(s_{1}(t), \ldots, s_{m}(t)\right)^{T}$ of the springs converge, as $t \rightarrow \infty$, to the attractor

$$
S(t)=A(X(t)-h(t)+g(t))
$$

where $X(t)$ is the set of all T-periodic solutions of sweeping process (3.11), and $h(t)$ and $g(t)$ are the effective loadings given by (3.9) and (3.8). The functions of $S(t)$ have equal derivatives for a.a. $t \geqslant 0$ as per (4.9) and, moreover,

$$
J_{C}\left(\bar{s}_{j}(t)\right)=J_{C}\left(\hat{s}_{j}(t)\right), \quad \text { for all } \bar{s}, \hat{s} \in \operatorname{ri}(S), t \geqslant 0
$$


By analogy with Theorem 4.8 , the meaning of $\operatorname{ri}(S)$ is

$$
\operatorname{ri}(S)=\{s \in S: s(0) \in \operatorname{ri}(S(0))\} .
$$

Proof. We apply Theorem 4.8 with $C(t)=\Pi(t) \cap V$, where $\Pi(t)$ and $V$ are those defined in Theorem 3.1. Since $\Pi(t)$ is uniformly bounded in $t \in[0, T]$, same holds for $C(t)$. Thus, the conditions of Theorem 4.8 are satisfied with $c_{j}^{-}(t)=c_{j}^{-}+a_{j} h_{j}(t)$ and $c_{j}^{+}(t)=c_{j}^{+}+a_{j} h_{j}(t)$, and Theorem 4.8 implies that

$$
J\left(t, A^{-1} \bar{s}(t)+h(t)-g(t)\right)=J\left(t, A^{-1} \hat{s}(t)+h(t)-g(t)\right), \quad \text { for all } \bar{s}, \hat{s} \in \operatorname{ri}(S), t \geqslant 0,
$$

which equivalent formulation is (4.26). Other statements of Theorem 4.10 follow from Theorem 4.8 just directly. The proof of the theorem is complete.

Property (4.26) says that, for any $j \in \overline{1, m}$, the spring $i$ will asymptotically execute a certain pattern of elastic deformation which doesn't depend on the state of the network at the initial time.

Example (continued). For the elastoplastic system (2.1)-(2.5) of Figure 6 with $T$-periodic displacementcontrolled and stress-controlled loadings $l(t)$ and $h(t)$, Theorem 4.10 implies the convergence of stresses $s(t)$ to a $T$-periodic attractor $S(t)$ provided that property (3.43) holds. Furthermore, the functions of $A^{-1} S(t)+h(t)-g(t)$ are all non-constant, if (3.51) is satisfied. In the next section of the paper we offer a general result which will, in particular, imply that the attractor $A^{-1} S(t)+h(t)-g(t)$ consists of a single solution.

\section{Stabilization to a unique nON-Stationary Periodic SOlution}

In this section we first prove that the periodic attractor $X(t)$ of a general sweeping process (4.1) in a vector space $E$ of dimension $d$ consists of just one non-stationary $T$-periodic solution, when the normals of any $d$ different facets of the moving polyhedron $C(t)$ are linearly independent. Then we give a sufficient condition for such a requirement to hold for the sweeping process (3.11) coming from the elastoplastic system (2.1)-(2.5).

\subsection{Stabilization of a general sweeping process with a polyhedral moving set}

As earlier, let $E$ be a linear vector space of dimension $d$ and let $(\cdot, \cdot)_{0}$ be a scalar product in $E$. In this subsection it will be convenient to rewrite the set (4.20) in the following form

$$
C(t)=\bigcap_{j=1}^{M}\left\{x \in E:\left(x, n_{j}\right)_{0} \leqslant c_{j}(t)\right\}, \quad t \geqslant 0,
$$

where $c_{j}$ are single-valued functions and $n_{j}$ are given vectors of $E$. The advantage of form (5.1) compared to (4.20) is that any vector of $N_{C(t)}^{0}(x)$ has non-negative coordinates in the basis formed by the normals $n_{1}, \ldots, n_{M}$. Then we establish the following result about the global asymptotic stability of sweeping processes.

Theorem 5.1. Let $t \mapsto C(t)$ be a uniformly bounded set-valued function given by (5.1), where the functions $c_{i}$ are globally Lipschitz continuous and T-periodic, and $M \geqslant \operatorname{dim} E$. Assume that any $\operatorname{dim} E$ vectors out of the collection $\left\{n_{j}\right\}_{j=1}^{M} \subset E$ are linearly independent and the cardinality of the set

$$
J(t, x)=\left\{i \in \overline{1, M}:\left(x, n_{j}\right)_{0}=c_{j}(t)\right\}
$$

doesn't exceed $\operatorname{dim} E$ for all $x \in C(t)$ and $t \in[0, T]$. Then the set $X(t)$ of $T$-periodic solutions of sweeping process (4.1) contains at most one non-constant T-periodic solution.

Note, $n_{j}$ in (5.1) are, generally speaking, different from $n_{j}$ in (4.20), but we use same notation as it shouldn't cause confusion. Accordingly, the active set $J(t, x)$ of Theorem 5.1 is different from the active set $J(t, x)$ of Theorem 4.8. 
Lemma 5.2. Assume, that for each $t \in[0, T]$ and $x \in C(t)$ the collection of vectors $\left\{n_{j}: j \in J(t, x)\right\}$ is linearly independent. Then for a solution $x(t)$ of sweeping process (4.1) there is a collection of integrable non-negative $\lambda_{j}:[0, T] \rightarrow[0, \infty), j \in \overline{1, M}$, such that

$$
-\dot{x}(t)=\sum_{j=1}^{M} \lambda_{j}(t) n_{j}, \quad \text { for a.a. } t \in[0, T] .
$$

Proof. Recall, that for a fixed $t \in[0, T]$, the normal cone (4.2) to the set $C(t)$ of polyhedral form (5.1) can be equivalently formulated as (see e.g. Hiriart-Urruty and Lemaréchal [26], Ex. 5.2.6, p. 67)

$$
N_{C(t)}^{0}(x)= \begin{cases}\{0\}, & \text { if } x \in \operatorname{int} C(t), \\ \left\{\left(\sum_{j \in J(t, x)} \lambda_{j} n_{j}\right): \lambda_{j} \geqslant 0\right\}, & \text { if } x \in \partial C(t), \\ \emptyset, & \text { if } x \notin C(t) .\end{cases}
$$

Here int $C(t)$ and $\partial C(t)$ are respectively the interior and the boundary of $C(t)$. Therefore, for a.a. fixed $t \in[0, T]$, the existence of $\lambda(t) \in \mathbb{R}^{M}, \lambda_{j}(t) \geqslant 0, j \in \overline{1, M}$, verifying

$$
-\dot{x}(t)=\sum_{j \in J(t, x(t))} \lambda_{j}(t) n_{j}
$$

follows from the inclusion (4.1). We set $\lambda_{j}(t)=0$, if $j \notin J(t, x(t))$. The proof of Lebesgue measurability of $\lambda(t)$ will be split into several steps.

Step 1. First we observe that, for any $\hat{t} \in[0, T]$,

$$
\text { the set } T_{\hat{t}}=\{t \in[0, T]: J(t, x(t))=J(\hat{t}, x(\hat{t}))\} \text { is measurable. }
$$

This follows from the fact that the set $\left\{t \in[0, T]:\left\langle x(t), n_{j}(t)\right\rangle-c_{j}(t)=0\right\}$ is measurable for each fixed index $j \in \overline{1, M}$ and that $J(\hat{t}, x(\hat{t})) \subset \overline{1, M}$.

Step 2. Now we fix some $\hat{t} \in[0, T]$ and prove that, for any Borel set $B \subset \mathbb{R}^{M}$,

$$
\text { the set } T_{\hat{t}}(B)=\{t \in[0, T]: \lambda(t) \in B, J(t, x(t))=J(\hat{t}, x(\hat{t}))\} \text { is measurable. }
$$

If inclusion (4.1) doesn't hold at $\hat{t}$ and $\operatorname{mes}\left(T_{\hat{t}}\right)=0$, then $T_{\hat{t}}(B)$ is measurable and mes $\left(T_{\hat{t}}(B)\right)=0$. If $(4.1)$ doesn't hold at $\hat{t}$ and $\operatorname{mes}\left(T_{\hat{t}}\right)>0$, then we can find $\tilde{t} \in T_{\hat{t}}$ such that (4.1) does hold at $\tilde{t}$. Since $T_{\hat{t}}=T_{\tilde{t}}$, we conclude that one won't restrict generality of the proof, if assume that (4.1) holds for the initially chosen $\hat{t} \in[0, T]$.

Let $\bar{n}_{1}, \ldots, \bar{n}_{d}$ be any basis in $E$ such that

$$
\bar{n}_{j}=n_{j}, \quad \text { for all } j \in J(\hat{t}, x(\hat{t})),
$$

therefore it depends on $\hat{t}$. Denote by $S_{\hat{t}}: E \rightarrow \mathbb{R}^{d}$ the bounded linear map which maps every vector from $E$ to its coordinates in terms of $\left\{\bar{n}_{j}\right\}_{j=1}^{d}$. Then (5.4) necessarily means that

$$
\lambda(t)=-S_{\hat{t}} \dot{x}(t), \quad \text { for a.a. } t \in[0, T] \text { such that } J(t, x(t))=J(\hat{t}, x(\hat{t})) .
$$


Therefore, up to a subset of $[0, T]$ of zero measure,

$$
T_{\hat{t}}(B)=\left\{t \in[0, T]:-S_{\hat{t}} \dot{x}(t) \in B, J(t, x(t))=J(\hat{t}, x(\hat{t}))\right\}=\left(-S_{\hat{t}} \dot{x}\right)^{-1}(B) \cap T_{\hat{t}},
$$

and the measurability of $T_{\hat{t}}(B)$ follows by combining the continuity of $x$ and the conclusion of Step 1 .

Step 3. We finally fix a Borel set $B \subset \mathbb{R}^{k}$ and prove the measurability of the set

$$
\lambda^{-1}(B)=\{t \in[0, T]: \lambda(t) \in B\} .
$$

Since $J(\hat{t}, x(\hat{t}))$ can take only a finite number of (set-valued) values when $\hat{t}$ varies from 0 to $T$, then there is a finite sequence $t_{1}, \ldots, t_{K} \in[0, T]$ such that

$$
[0, T]=\bigcup_{i \in \overline{1, K}} T_{t_{i}}
$$

and so we can rewrite (5.5) as follows

$$
\lambda^{-1}(B)=\bigcup_{i \in \overline{1, K}} T_{t_{i}}(B)
$$

which is a finite union of measurable sets. The proof of the measurability of $\lambda$ is complete.

The integrability of $\lambda$ on $[0, T]$ now follows from its boundedness. Indeed, since, $\|\dot{x}(t)\|_{0} \leqslant L$ for a.a. $t \in[0, T]$ and some $L>0$ ([38], p. 13), one has

$$
\left|\lambda_{i}(t)\right| \leqslant\|\lambda(t)\|=L \max _{i \in \overline{1}, K}\left\|S_{t_{i}}\right\|, \quad \text { for a.a. } t \in[0, T] .
$$

The proof of the lemma is complete.

Proof of Theorem 5.1. Let $x(t)$ and $y(t)$ be two non-constant distinct $T$-periodic solutions of (3.11). Theorem 4.8 implies that we won't lose generality by assuming that

$$
J(t, x(t))=J(t, y(t)) .
$$

When applying Theorem 4.8 we used the fact that the set (5.1) can be expressed in the form (4.20) due to the uniform boundedness of $C(t)$.

The proof is by reaching a contradiction with the fact that $x(t)$ and $y(t)$ are distinct.

By replacing $-\dot{x}(t)$ by its representation from Lemma 5.2 and using $T$-periodicity of $x(t)$, one gets

$$
0=\int_{0}^{T}-\dot{x}(t) \mathrm{d} t=\int_{0}^{T} \sum_{i=1}^{M} \lambda_{j}(t) n_{j} \mathrm{~d} t=\sum_{i=1}^{M} \int_{0}^{T} \lambda_{j}(t) \mathrm{d} t n_{j},
$$

where $\lambda_{j}(t) \geqslant 0$. Since $x(t)$ is non-constant and the moving constraint $C(t)$ is the only force that influences the value of $\dot{x}(t)$, the set

$$
\hat{J}:=\left\{j \in \overline{1, M}: \int_{0}^{T} \lambda_{j}(t) \mathrm{d} t>0\right\}
$$

is non-empty. The following two cases can take place. 
1) $\left\{n_{j}: j \in \hat{J}\right\}$ is a linearly independent system. But property (5.7) yields

$$
\sum_{j \in \hat{J}} \int_{0}^{T} \lambda_{j}(t) \mathrm{d} t n_{j}=0
$$

that, for linearly independent vectors $n_{j}$, can happen only when $\int_{0}^{T} \lambda_{j}(t) \mathrm{d} t \equiv 0, j \in \overline{1, M}$. Therefore case 1$)$ cannot take place as $x(t)$ is non-constant.

2) The vectors of $\left\{n_{j}: j \in \hat{J}\right\}$ are linearly dependent. Since, by the assumption of the theorem, any $d$ vectors from $\left\{n_{j}: j \in \hat{J}\right\}$ are linearly independent, one must have $|\hat{J}|>d$. Let us show this leads to a contradiction as well.

Since for each $j_{*} \in \hat{J}$, the function $\lambda_{j_{*}}(t)$ is positive on a set of positive measure, there are time moments $\left\{t_{j_{*}}\right\}_{j_{*} \in \hat{J}}$, where (5.6) holds along with

$$
-\dot{x}\left(t_{i}\right)=\sum_{j=1}^{M} \lambda_{j}\left(t_{i}\right) n_{j} \quad \text { and } \quad \lambda_{i}\left(t_{i}\right)>0 .
$$

This and (5.3) imply

$$
i \in J\left(t_{i}, x\left(t_{i}\right)\right) \quad \text { and by }(5.6) \quad i \in J\left(t_{i}, y\left(t_{i}\right)\right), \quad i \in \hat{J}
$$

or, equivalently,

$$
\left(x\left(t_{i}\right), n_{i}\right)_{0}=c_{i}\left(t_{i}\right) \quad \text { and } \quad\left(y\left(t_{i}\right), n_{i}\right)_{0}=c_{i}\left(t_{i}\right), \quad i \in \hat{J} .
$$

Therefore,

$$
\left(x\left(t_{i}\right)-y\left(t_{i}\right), n_{i}\right)_{0}=0, \quad i \in \hat{J},
$$

and, by Lemma 4.5 ,

$$
\left(x(0)-y(0), n_{i}\right)_{0}=0, \quad i \in \hat{J} .
$$

But $|\hat{J}|>d$ and so $\left\{n_{j}: j \in \hat{J}\right\}$ contains $d$ linearly independent vectors, which form a basis of $\mathbb{R}^{d}$. Therefore, $x(0)=y(0)$, which is a contradiction.

Theorem 5.1 can be used for stabilization of general sweeping process with polyhedral moving set such as those considered e.g. in Colombo et al. [16] and Krejci-Vladimirov [37].

A fundamental case where Theorem 5.1 allows to stabilize an elastoplastic system (2.1)-(2.5) to a single periodic solution is when $V$ cut $\Pi(t)$ along a simplex. Indeed, the corollary below follows by observing that the conditions of Theorem 5.1 about the number of facets $k$ and about the cardinality of the set $J(t, x)$ always hold when $C(t)$ is a simplex and regardless of whether $C(t)$ is expressed in the form (4.20) or in the form (5.1).

Corollary 5.3. Let $t \mapsto C(t)$ be a uniformly bounded set-valued function given by (4.20), where the functions $c_{i}(t)$ are globally Lipschitz continuous and T-periodic. If $C(t)$ is a simplex for any $t \in[0, T]$, then the set $X(t)$ of T-periodic solutions of sweeping process (4.1) contains at most one non-constant T-periodic solution. 
Testing the set $\Pi(t) \cap V$ for being a simplex can be executed for any given elastoplastic system (2.1)-(2.5) using the algorithms of computational geometry (e.g. Bremner et al. [7] can be used to compute the vertexes of $\Pi(t) \cap V$ whose number needs to equal $m+1)$.

At the same time, establishing analytic criteria for stabilization to occur could be of great use in materials science. A simple criterion of this type is offered in the following section of the paper.

\subsection{Application: an analytic condition for stabilization of elastoplastic systems to a unique periodic regime}

The following theorem is the main result of this paper. It can be viewed as an analogue of high gain feedback stabilization in control theory. Indeed, one of the two central assumptions of the theorem is $q=n-2$, which means that the elastoplastic system has a sufficient number of control variables to be fully controllable and thus stabilizable. The second central assumption is assuming that the magnitude of the stress-controlled loading is high enough which literally resembles the high gain requirement of feedback control theory.

The idea of Theorem 5.4 is based on a simple fact that the moving parallelepiped $\Pi(t)$ intersects the plane $V$ along a simplex, if the plane $V$ is close to the vertex of the parallelepiped, see Figure $7 \mathrm{~d}$. At the same time, this geometric statement turned out to hold only if $q=n-2$.

Theorem 5.4. In the settings of Proposition 3.12 assume that the stress-controlled loading $h(t)$ satisfies

$$
\left\langle\bar{u},\left(\begin{array}{c}
\bar{c}_{1}^{\alpha} \\
\vdots \\
\bar{c}_{j-1}^{\alpha} \\
\bar{c}_{j}^{-\alpha} \\
\bar{c}_{j+1}^{\alpha} \\
\vdots \\
\bar{c}_{m}^{\alpha}
\end{array}\right)+A h(t)\right\rangle \cdot\left\langle\bar{u}, \bar{c}^{\alpha}+A h(t)\right\rangle \leqslant 0, \quad j \in \overline{1, m}, t \in[0, T]
$$

for at least one $\alpha \in\{-1,+1\}$. Further assume that the amplitude of the displacement-controlled loading $g(t)$ is large in the sense of (3.44). If both $h(t)$ and $g(t)$ are T-periodic and globally Lipschitz continuous, then there exists a T-periodic function $s_{0}(t)$ such that $\left\|s(t)-s_{0}(t)\right\| \rightarrow 0$ as $t \rightarrow \infty$ for the stress component $s(t)$ of any solution of the quasistatic evolution problem (2.1)-(2.5).

Remark 5.6 below explains that, in the important case where the elasticity bounds of all springs are symmetric about 0 , condition (5.8) is a requirement for $h(t)$ to be large enough, but not too large.

Remark 5.5. Following the lines of Remark 3.13, we consider the left-hand-side of (5.8) as a polynomial $P(\langle\bar{u}, A h(t)\rangle)$ in $\langle\bar{u}, A h(t)\rangle$, so that the branches of the polynomial are pointing upwards. Therefore, condition (5.8) is the requirement for $\langle\bar{u}, A h(t)\rangle$ to stay between the roots of the polynomial. Note, one root of $P(\langle\bar{u}, A h(t)\rangle)$ is given by $\langle\bar{u}, A h(t)\rangle=-\left\langle\bar{u}, \bar{c}^{\alpha}\right\rangle$. By computing the derivative $P^{\prime}\left(-\left\langle\bar{u}, \bar{c}^{\alpha}\right\rangle\right)$ one concludes that $\langle\bar{u}, A h(t)\rangle=$ $-\left\langle\bar{u}, \bar{c}^{\alpha}\right\rangle$ is the smaller or larger root of $P(\langle\bar{u}, A h(t)\rangle)$ according to whether $\alpha=+1$ or $\alpha=-1$. Therefore, a 
sufficient condition for (5.8) to hold with $\alpha=+1$ and $\alpha=-1$ are

$$
\left\langle\bar{u},-\bar{c}^{+}\right\rangle \leqslant\langle\bar{u}, A h(t)\rangle \leqslant \min _{j \in \overline{1, m}}\left\langle\bar{u},-\left(\begin{array}{c}
\bar{c}_{1}^{+} \\
\vdots \\
\bar{c}_{j-1}^{+} \\
\bar{c}_{j}^{-} \\
\bar{c}_{j+1}^{+} \\
\vdots \\
\bar{c}_{m}^{+}
\end{array}\right)\right\rangle
$$

and

$$
\max _{j \in \overline{1, m}}\left\langle\bar{u},-\left(\begin{array}{c}
\bar{c}_{1}^{-} \\
\vdots \\
\bar{c}_{j-1}^{-} \\
\bar{c}_{j}^{+} \\
\bar{c}_{j+1}^{-} \\
\vdots \\
\bar{c}_{m}^{-}
\end{array}\right)\right\rangle \leqslant\langle\bar{u}, A h(t)\rangle \leqslant\left\langle\bar{u},-\bar{c}^{-}\right\rangle
$$

respectively. As we show in the proof, conditions (5.9) and (5.10) ensure that $V$ cuts $\Pi(t)$ either along the edges adjacent to the $A^{-1} \bar{c}^{+}+h(t)-g(t)$ vertex of $\Pi(t)$ or along the edges adjacent to the $A^{-1} \bar{c}^{-}+h(t)-g(t)$ vertex of $\Pi(t)$. In particular (5.9)-(5.10) rule out the possibility of cross-section $C(t)$ of Figure 3 and allow for cross-section $C\left(t_{1}\right)$ of the same figure.

Remark 5.6. We note, that conditions (5.9)-(5.10) never hold for $h(t)=0$ as long as the elastic bounds are symmetric in the sense that

$$
\left[c_{i}^{-}, c_{i}^{+}\right]=\left[-c_{i}, c_{i}\right] \quad \text { and } \quad c_{i}>0, \quad i \in \overline{1, m}
$$

which is often the case in materials science applications (see e.g. Holmes [27], Fig. B.9). Therefore, when the symmetry condition (5.11) holds, the inequality (5.8) is a requirement for $h(t)$ to be distant from 0 , but not too distant.

Indeed, let us show that, under condition (5.11), the right-hand-side of (5.9) is always negative when $m=3$ (the general case can be addressed by analogy). Property (5.11) implies that

$$
\bar{c}^{+}=\left(\begin{array}{c}
\operatorname{sign}\left(\bar{u}_{1}\right) c_{1} \\
\operatorname{sign}\left(\bar{u}_{2}\right) c_{2} \\
\operatorname{sign}\left(\bar{u}_{3}\right) c_{3}
\end{array}\right), \quad \bar{c}^{-}=\left(\begin{array}{c}
-\operatorname{sign}\left(\bar{u}_{1}\right) c_{1} \\
-\operatorname{sign}\left(\bar{u}_{2}\right) c_{2} \\
-\operatorname{sign}\left(\bar{u}_{3}\right) c_{3}
\end{array}\right),
$$

so that the right-hand-side of (5.9) takes the form

$$
Q=\min \left\{\left|u_{1}\right| c_{1}-\left|u_{2}\right| c_{2}-\left|u_{3}\right| c_{3},-\left|u_{1}\right| c_{1}+\left|u_{2}\right| c_{2}-\left|u_{3}\right| c_{3},-\left|u_{1}\right| c_{1}-\left|u_{2}\right| c_{2}+\left|u_{3}\right| c_{3}\right\} .
$$

Fix some order of the numbers $\left|u_{1}\right| c_{1},\left|u_{2}\right| c_{2},\left|u_{3}\right| c_{3}$, e.g. consider

$$
\left|u_{1}\right| c_{1} \leqslant\left|u_{3}\right| c_{3} \leqslant\left|u_{2}\right| c_{2}
$$


(the case of an arbitrary order can be dealt with by analogy). First, by (5.13) we have $\left|u_{1}\right| c_{1}-\left|u_{3}\right| c_{3} \leqslant 0$, therefore

$$
\min \left\{\left|u_{1}\right| c_{1}-\left|u_{3}\right| c_{3},-\left|u_{1}\right| c_{1}+\left|u_{3}\right| c_{3}\right\}=\left|u_{1}\right| c_{1}-\left|u_{3}\right| c_{3},
$$

which allows to drop the 3rd term in (5.12) obtaining

$$
Q=\min \left\{\left|u_{1}\right| c_{1}-\left|u_{2}\right| c_{2}-\left|u_{3}\right| c_{3},-\left|u_{1}\right| c_{1}+\left|u_{2}\right| c_{2}-\left|u_{3}\right| c_{3}\right\} .
$$

Now we use (5.13) again to observe that $\left|u_{1}\right| c_{1}-\left|u_{2}\right| c_{2} \leqslant 0$, therefore

$$
\min \left\{\left|u_{1}\right| c_{1}-\left|u_{2}\right| c_{2},-\left|u_{1}\right| c_{1}+\left|u_{2}\right| c_{2}\right\}=\left|u_{1}\right| c_{1}-\left|u_{2}\right| c_{2},
$$

and so to drop the second term in (5.14) obtaining

$$
Q=\left|u_{1}\right| c_{1}-\left|u_{2}\right| c_{2}-\left|u_{3}\right| c_{3},
$$

which is negative by (5.13). In the general case of $m$ springs, the inequalities of (5.13) will successively reduce the expression for $Q$ (which is a min of $m$ terms initially) until only one term remains. This single term will consist of the first term of (5.13) with positive sign and the rest of the terms of (5.13) with negative signs, so that this single term will be always negative by (5.13), as in (5.15).

Similarly, the left-hand-side in (5.10) is always positive, if the symmetry condition (5.11) holds.

The interested reader is referred to $e . g$. Grzesikiewicz et al. [22] for an elastoplastic system where the symmetry condition (5.11) doesn't hold (Shape Memory Alloys).

Proof of Theorem 5.4. We are going to prove that, for the sweeping process (3.30) of the elastoplastic system given, the moving constraint (3.29) is a simplex, so that the conclusion will follow by applying Theorem 4.10 and Corollary 5.3.

Since the set

$$
C(t)=\bigcap_{j=1}^{m}\left\{x \in V: c_{j}^{-}+a_{j} h_{j}(t) \leqslant a_{j} x_{j} \leqslant c_{j}^{+}+a_{j} h_{j}(t)\right\}
$$

is just a parallel displacement of the polyhedron (3.29), the proof will be complete, if we establish that $C(t)$ given by (5.16) is a simplex.

Fix $t \in[0, T]$. In what follows, we show that $C(t)=\operatorname{conv}\left\{\xi^{i}, i \in \overline{1, m}\right\}$, where the vertices $\xi^{1}, \ldots, \xi^{m}$ are given by the equations

$$
\begin{aligned}
& \left\langle\bar{u}, A \xi^{i}\right\rangle=0, \\
& a_{j} \xi_{j}^{i}=\bar{c}_{j}^{\alpha}+a_{j} h_{j}(t), \quad j \in \overline{1, m}, i \neq j .
\end{aligned}
$$

The solution $\xi^{i}$ is unique because none of the components of $\bar{u}$ vanish as follows from Lemma 3.11. To clarify the meaning of (5.17)-(5.18) we recall that assuming (5.18) for all $j \in \overline{1, m}$ (including $i=j$ ) would mean that $\xi_{j}=A^{-1} \bar{c}_{j}^{\alpha}+h_{j}(t)$. Therefore, the presence of $i \neq j$ in (5.18) allows $\xi_{j}$ to vary along one of the edges of $A^{-1} C+h_{j}(t)$ adjacent to the vertex $A^{-1} \bar{c}_{j}^{\alpha}+h_{j}(t)$. Among all such locations of $\xi_{j}$, condition (5.17) selects the one for which $\xi_{j} \in V$.

In our proof we will distinguish two cases: when the property

$$
\left\langle\bar{u}, A\left(A^{-1} \bar{c}^{\alpha}+h(t)\right)\right\rangle=0
$$


holds and when the property (5.19) doesn't hold. Let us show that (5.19) implies that $C(t)$ is the singleton $C(t)=\left\{A^{-1} \bar{c}^{\alpha}+h(t)\right\}$. Indeed, assume that there exists $x \in C(t)$ such that $x \neq A^{-1} \bar{c}^{\alpha}+h(t)$. Then $x$ can be expressed as $x=A^{-1} c+h(t)$ for some $c \in C$. On the other hand, $x \in V$ implies $\langle\bar{u}, A x\rangle=0$. Therefore, $\left\langle\bar{u}, \bar{c}^{\alpha}-c\right\rangle=0$ and by just expanding the scalar product we get the existence of two indices $j_{1}, j_{2} \in \overline{1, m}$, such that

$$
\bar{u}_{j_{1}}\left(\bar{c}_{j_{1}}^{\alpha}-c_{j_{1}}\right)>0, \quad \bar{u}_{j_{2}}\left(\bar{c}_{j_{2}}^{\alpha}-c_{j_{2}}\right)<0
$$

It remains to observe that (5.20) can never hold by the construction of $\bar{c}^{\alpha}$ (see formulation of Prop. 3.12), that is seen by listing all possible cases as follows:

$$
\begin{gathered}
\text { if } \quad \alpha=-1, \bar{u}_{j}<0, \text { then } \bar{u}_{j}\left(\bar{c}_{j}^{\alpha}-c_{j}\right)=\bar{u}_{j}\left(c_{j}^{+}-c_{j}\right) \leqslant 0, \\
\text { if } \quad \alpha=-1, \bar{u}_{j}>0, \text { then } \bar{u}_{j}\left(\bar{c}_{j}^{\alpha}-c_{j}\right)=\bar{u}_{j}\left(c_{j}^{-}-c_{j}\right) \leqslant 0, \\
\text { if } \quad \alpha=1, \bar{u}_{j}<0, \text { then } \bar{u}_{j}\left(\bar{c}_{j}^{\alpha}-c_{j}\right)=\bar{u}_{j}\left(c_{j}^{-}-c_{j}\right) \geqslant 0, \\
\text { if } \quad \alpha=1, \bar{u}_{j}>0, \text { then } \bar{u}_{j}\left(\bar{c}_{j}^{\alpha}-c_{j}\right)=\bar{u}_{j}\left(c_{j}^{+}-c_{j}\right) \geqslant 0,
\end{gathered}
$$

where we also used that $c_{j}^{-} \leqslant c_{j} \leqslant c_{j}^{+}, j \in \overline{1, m}$. This list of cases shows that opposite signs in the two inequalities of (5.20) can never occur. Therefore, if (5.19) holds, then $C(t)$ is a singleton. But if $C(t)$ is a singleton for at least one $t_{*} \in[0, T]$, then the statement of the theorem becomes trivial (all the solutions will stick to a single solution at time $t_{*}$ ). That is why we now focus on the case where (5.19) doesn't hold anywhere on $[0, T]$. Assuming that (5.19) doesn't hold anywhere on $[0, T]$, we will prove that $C(t)$ is a simplex given by $C(t)=\operatorname{conv}\left\{\xi^{i}, i \in \overline{1, m}\right\}$. Step 1: It holds $\xi^{i} \in C(t), i \in \overline{1, m}$. Based on formula (5.16), we have to show that

$$
c_{i}^{-}+a_{i} h_{i}(t) \leqslant a_{i} \xi_{i}^{i} \leqslant c_{i}^{+}+a_{i} h_{i}(t), \quad i \in \overline{1, m} .
$$

Fix $i \in \overline{1, m}$ and consider the function

$$
b(x)=\left\langle\bar{u},\left(\begin{array}{c}
\bar{c}_{1}^{\alpha} \\
\vdots \\
\bar{c}_{i-1}^{\alpha} \\
x \\
\bar{c}_{i+1}^{\alpha} \\
\vdots \\
\bar{c}_{m}^{\alpha}
\end{array}\right)+\left(\begin{array}{c}
a_{1} h_{1}(t) \\
\vdots \\
a_{i-1} h_{i-1}(t) \\
0 \\
a_{i+1} h_{i+1}(t) \\
\vdots \\
a_{m} h_{m}(t)
\end{array}\right)\right\rangle .
$$

By the definition, $a_{i} \xi_{i}^{i}$ is the unique root of the equation $b(x)=0$. On the other hand, condition (5.8) implies that $b\left(\bar{c}_{i}^{-k}+a_{i} h_{i}(t)\right) \cdot b\left(\bar{c}_{i}^{\alpha}+a_{i} h_{i}(t)\right) \leqslant 0$, so that the unique zero of $b(x)$ must be located between the numbers $\bar{c}_{i}^{-\alpha}+a_{i} h_{i}(t)$ and $\bar{c}_{i}^{\alpha}+a_{i} h_{i}(t)$.

Step 2: The vertices $\xi^{i}, i \in \overline{1, m}$, form an $m-1$-simplex. For a given $i \in \overline{1, m}$, we need to show that $m-1$ vectors

$$
\zeta^{j i}=\xi^{j}-\xi^{i}, \quad j \in \overline{1, m}, i \neq j,
$$

are linearly independent. From (5.17) we have

$$
\left\langle\bar{u}, A \zeta^{j i}\right\rangle=0
$$


while from (5.18) we get

$$
\zeta^{j i}=\left(0, \ldots, 0, \zeta_{j}^{j i}, 0, \ldots, 0, \zeta_{i}^{j i}, 0, \ldots, 0\right)^{T}
$$

Combining these two properties we conclude that

$$
u_{j} a_{j} \zeta_{j}^{j i}+u_{i} a_{i} \zeta_{i}^{j i}=0
$$

By Lemma $3.11 u_{j} a_{j} \neq 0$ and $u_{i} a_{i} \neq 0$, therefore we either have $\zeta_{j}^{j i}=\zeta_{i}^{j i}=0$ or $\zeta_{j}^{j i} \zeta_{i}^{j i} \neq 0$. Observe that the former case is impossible. Indeed, if $\xi^{i_{1}}=\xi^{i_{2}}$ for some $i_{1} \neq i_{2}$, then (5.18) implies $\xi^{i_{1}}=\xi^{i_{2}}=A^{-1}\left(\bar{c}^{\alpha}+A h(t)\right)$, which leads to (5.19) when plugged to (5.17) which we already excluded.

It remains to notice that property $\zeta_{j}^{j i} \zeta_{i}^{j i} \neq 0, i \neq j$ implies that the vectors (5.22) are linearly independent through $i \neq j, j \in \overline{1, m}$.

Step 3: We claim that $C(t)=\operatorname{conv}\left\{\xi^{i}, i \in \overline{1, m}\right\}$. From Step 1, $C(t) \supset \operatorname{conv}\left\{\xi^{i}, i \in \overline{1, m}\right\}$, so it remains to prove that $C(t) \subset \operatorname{conv}\left\{\xi^{i}, i \in \overline{1, m}\right\}$.

We fix $\hat{i} \in \overline{1, m}$ and consider a facet conv $\left\{\xi^{j}, j \neq \hat{i},\right\}$ of the simplex $\operatorname{conv}\left\{\xi^{j}, j \in \overline{1, m}\right\}$. Observe from (5.18) that all vertices of the facet share their $i$ th coordinate. Therefore the whole facet belongs to the plane

$$
L^{\hat{i}}=\left\{x \in V: a_{i} x_{i}=\bar{c}_{\hat{i}}^{k}+a_{\hat{i}} h_{\hat{i}}(t)\right\}
$$

Therefore,

$$
\operatorname{conv}\left\{\xi^{j}, j \in \overline{1, m}\right\}=\bigcap_{i=1}^{m}\left\{x \in V: p_{i}\left(a_{i} x_{i}-\bar{c}_{i}^{k}-a_{i} h_{i}(t)\right) \leqslant 0\right\},
$$

where $p_{i} \in\{-1,1\}$ are suitable signs. On the other hand, by (5.16),

$$
C(t) \subset \bigcap_{i=1}^{m}\left\{x \in V: q_{i}\left(a_{i} x_{i}-\bar{c}_{i}^{\alpha}-a_{i} h_{i}(t)\right) \leqslant 0\right\},
$$

where $q_{i} \in\{-1,1\}$ are suitable signs. Since by Step $2, \operatorname{conv}\left\{\xi_{i}, i \in \overline{1, m}\right\} \subset C(t)$, we get $p_{i}=q_{i}, i \in \overline{1, m}$. But then (5.23) takes the form $C(t) \subset \operatorname{conv}\left\{\xi_{i}, i \in \overline{1, m}\right\}$.

To summarize, we proved that, when (5.19) doesn't hold, the set $C(t)$ is a simplex and the conclusion follows by applying Proposition 3.14, Remark 4.1, Theorem 4.10 and Corollary 5.3. Specifically, the attractor $S(t)$ that is introduced in the formulation of Theorem 4.10 is non-empty by Remark 4.1. Corollary 5.3 then implies that the set of $T$-periodic functions $S(t)$ contains at most one non-constant $T$-periodic solution. On the other hand, Proposition 3.14 says that $S(t)$ cannot contain constant solutions. Therefore, $S(t)$ consists of just one $T$-periodic function, that we denote by $s_{0}$. Finally, Theorem 4.10 ensures that $\left\|s(t)-s_{0}(t)\right\| \rightarrow 0$ as $t \rightarrow \infty$ for any stress component $s(t)$ (that we call "stress-vector" in the formulation of Thm. 4.10) of any solution of the quasistatic evolution problem (2.1)-(2.5).

Example (continued). Applying Theorem 5.4 to the elastoplastic system of Figure 6 (where we have $q=n-2$ ) we use earlier formulas (3.37) and (3.42) together with Remark 5.5 to obtain the following conclusion: if the $T$-periodic displacement-controlled loading $l(t)$ satisfies (3.51) and, for the $T$-periodic stress-controlled loading $h(t)$, one either has

$$
\begin{aligned}
-\bar{c}_{1}^{+} & +\bar{c}_{2}^{-}-\bar{c}_{3}^{+}<a_{1} h_{1}(t)+a_{2} h_{2}(t)+a_{3} h_{3}(t) \\
& <\min \left\{-\bar{c}_{1}^{-}+\bar{c}_{2}^{-}-c_{3}^{+},-\bar{c}_{1}^{+}+\bar{c}_{2}^{+}-\bar{c}_{3}^{+},-\bar{c}_{1}^{+}+\bar{c}_{2}^{-}-\bar{c}_{3}^{-}\right\}, \quad t \in[0, T],
\end{aligned}
$$


or

$$
\begin{aligned}
\max & \left\{-\bar{c}_{1}^{+}+\bar{c}_{2}^{+}-c_{3}^{-},-\bar{c}_{1}^{-}+\bar{c}_{2}^{-}-\bar{c}_{3}^{-},-\bar{c}_{1}^{-}+\bar{c}_{2}^{+}-\bar{c}_{3}^{+}\right\} \\
& <a_{1} h_{1}(t)+a_{2} h_{2}(t)+a_{3} h_{3}(t)<-\bar{c}_{1}^{-}+\bar{c}_{2}^{+}-\bar{c}_{3}^{-}, \quad t \in[0, T],
\end{aligned}
$$

then the stresses of springs of the elastoplastic system of Figure 6 converge, as $t \rightarrow \infty$, to a unique $T$-periodic regime that depends on $l(t)$ and $h(t)$, and doesn't depend on the initial state of the system.

\section{Conclusions}

We used Moreau sweeping process framework to analyze the asymptotic properties of quasistatic evolution of one-dimensional networks of elastoplastic springs (elastoplastic systems) under displacement-controlled and stress-controlled loadings. This type of elastoplastic systems covers, in particular, rheological models of materials science. We showed that displacement-controlled loading corresponds to parallel displacement of the moving polyhedron $C(t)$ of the respective sweeping process, but doesn't influence the shape of $C(t)$. We showed that it is the stress-controlled loading which is capable to change the shape of $C(t)$. Moreover, we proved that increasing the magnitude of the stress-controlled loading always makes $C(t)$ a simplex, if the number $q$ of displacement-controlled constraints is two less the number $n$ of nodes of the network $(q=n-2)$.

The global asymptotic stability result established in this paper ensures convergence of the stresses of springs to a unique periodic solution (output) when the magnitude of the displacement-controlled loading is large enough and when the normal vectors of any $d$ different facets of the moving polyhedron $C(t)$ are linearly independent. Here $d$ is the dimension of the phase space of the polyhedron $C(t)$, given by $d=m-n+q+1$, where $m$ is the number of springs, see (3.47). The most natural example where such a property holds is when $C(t)$ is a simplex.

In establishing our results we use monotonicity of the normal cone to say that the distance between any two solutions of a sweeping process doesn't increase over time, see Lemma 4.4 of Section 4.1 (we don't use maximal monotonicity of the normal cone explicitly). Geometric and algebraic properties of the normal cone that come from the polyhedral shape of the constraint play, however, the most crucial role in our proofs.

Our theory can be viewed as an analogue of the high gain feedback stabilization of the classical control theory, see [29], Section 4.7. The high gain assumption of the control theory corresponds to our condition (5.8) on the magnitude of stress-controlled loading. Our assumption $q=n-2$ on the network of elastoplastic springs resembles the relative degree in control.

The advantage of the proposed restriction $\operatorname{dim} U=1$ is that it leads to simple analytic conditions (3.44) and (5.8) for the convergence of an elastoplastic system, which can be used for the design of elastoplastic systems that converge for the desired set of applied loadings. Extending Theorem 5.4 to the case where $\operatorname{dim} U>1$ is a doable task, but the respective inequality (5.8) transforms into a list of groups of inequalities, where the number of groups equals the number of selections of $\operatorname{dim} U$ from $m$ (Eq. (5.17) gets replaced by the respective combinations of $\operatorname{dim} U$ equations). We don't see how such a condition can be useful in design of applied loadings, thus we stick to $\operatorname{dim} U=1$.

The results of the paper can be extended to the case of dynamic evolution of elastoplastic systems with small inertia forces along the lines of Martins et al. [43].

We like to think that the present paper opens a new room of opportunities for researchers interested in applied analysis and control.

\section{Appendix A. Derivation of system (2.1)-(2.5) from Moreau's ABSTRACT FRAMEWORK}

In this section we show how system (2.1)-(2.5) can be derived from the abstract framework by Moreau [45]. This abstract framework consists of the following 6 equations, that we quote directly from [45] keeping the numbering and placing the hat symbol "^" above Moreau's variables to distinguish them from the variables of 
the same name defined earlier in the present paper:

$$
\begin{aligned}
\hat{s} & =\hat{A} \hat{e}, \\
\dot{\hat{p}} & \in \partial \psi_{\hat{C}}(-\hat{f}), \\
\hat{\mathcal{L}} & =\hat{U}+\hat{g}(t), \\
-\hat{r} & \in \partial \psi_{\hat{\mathcal{L}}}(\hat{e}+\hat{p}), \\
\hat{r}+\hat{c}(t)-\hat{s} & =0, \\
\hat{s}+\hat{f} & =0,
\end{aligned}
$$

where $x \mapsto \psi_{B}(x)$ is the indicator function of a set $B$, whose subdifferential $\partial \psi_{B}(x)$ coincides with the normal cone $N_{B}(x)$ (see Rockafellar [50], p. 215). In [45], $\hat{U}+\hat{g}(t)$ is a perfect affine constraint, which moves according to $\hat{g}(t)$. The function $\hat{g}(t)$ is called guiding, and $\hat{c}(t)$ is called load.

Notice that $\partial \psi_{\hat{\mathcal{L}}}(\hat{e}+\hat{p})=N_{\hat{\mathcal{L}}}(\hat{p}+\hat{e})=\hat{U}^{\perp}=-\hat{U}^{\perp}$. Moreover, equation (6.4) necessarily means that $\hat{e}+\hat{p} \in \hat{\mathcal{L}}$ by definition of normal cone. Therefore system (6.1)-(6.6) can be equivalently written as

$$
\begin{aligned}
\hat{s} & =\hat{A} \hat{e}, \\
\dot{\hat{p}} & \in N_{\hat{C}}(\hat{s}), \\
\hat{e}+\hat{p} & \in \hat{U}+\hat{g}(t) \\
\hat{s}-\hat{c}(t) & \in \hat{U}^{\perp}
\end{aligned}
$$

To make a connection with the system (2.1)-(2.5) we plug the values of $e, p, s, A, U, g(t)$ and $-\bar{h}(t)$ (all defined in Sect. 2) as $\hat{e}, \hat{p}, \hat{s}, \hat{A}, \hat{U}, \hat{g}(t)$ and $\hat{c}(t)$ respectively into the system (6.1)-(6.2),(A.1)-(A.2) and we get

$$
\begin{aligned}
s & =A e, \\
\dot{p} & \in N_{C}(s), \\
e+p & \in U+g(t) \\
s+\bar{h}(t) & \in U^{\perp} .
\end{aligned}
$$

This is exactly the system (2.1)-(2.2),(3.20),(3.25) which is equivalent to the (2.1)-(2.5) as discussed in the proof of Theorem 3.1.

To summarize, the abstract framework (6.1)-(6.6) of Moreau [45] takes the form of (6.1)-(6.2) and (A.1)(A.2), which, in its turn, is equivalent to $(2.1)-(2.5)$ by formulas $(3.20),(3.25)$ when the appropriate variables are used.

\section{Appendix B. Some Results from graph theOry, Linear Algebra, CONVEX ANALYSIS}

This section contains some standard results that we use in the concepts and proofs of the paper. The notations of this section follow those of the original sources, which are Bapat [3], Friedberg et al. [20], Rockafellar [50], and Narici-Beckenstein [46].

Incidence matrix. A graph of $m$ oriented edges $\left\{e_{1}, \ldots, e_{m}\right\}$ on $n$ nodes $\{1, \ldots, n\}$ is described by an $n \times m$ incidence matrix $Q$ according to the following rule, see page 11 in [3]. The $i$ th raw of $Q^{T}$ contains only two non-zero elements " 1 " and " 1 ". The presence of " 1 " at $j$ th column of $i$ th raw means that the edge $e_{i}$ originates at note $j$. The presence of "- 1 " at $j$ th column of $i$ th raw means that edge $e_{i}$ terminates at note $j$. 


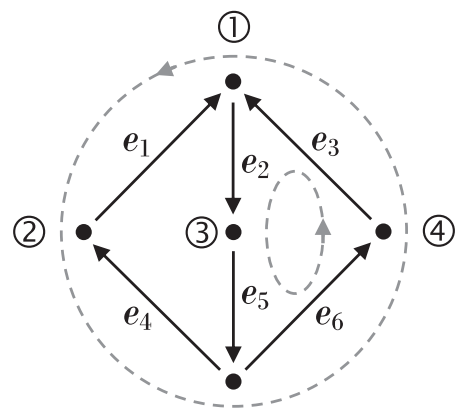

(5)

Figure B.1. An oriented graph of 6 edges on 5 nodes.

For example, for the graph of Figure B.1 (see Ex. 2.1 in [3]) the matrices $Q^{T}$ and $Q$ read as

$$
Q^{T}=\left(\begin{array}{ccccc}
-1 & 1 & 0 & 0 & 0 \\
1 & 0 & -1 & 0 & 0 \\
-1 & 0 & 0 & 1 & 0 \\
0 & -1 & 0 & 0 & 1 \\
0 & 0 & 1 & 0 & -1 \\
0 & 0 & 0 & -1 & 1
\end{array}\right), \quad Q=\left(\begin{array}{cccccc}
-1 & 1 & -1 & 0 & 0 & 0 \\
1 & 0 & 0 & -1 & 0 & 0 \\
0 & -1 & 0 & 0 & 1 & 0 \\
0 & 0 & 1 & 0 & 0 & -1 \\
0 & 0 & 0 & 1 & -1 & 1
\end{array}\right)
$$

Incidence vector, path, cycle. A finite sequence of successively connected edges $\left\{e_{j_{1}}, \ldots, e_{j_{k}}\right\}$ of the graph is called a oriented path, if each node of the graph connects at most two edges of the path. The $i$ th component of the incidence vector $x \in \mathbb{R}^{m}$ of the path is defined as $-1,0$, or 1 , according to whether the edge $e_{i}$ belongs to the path and disagrees with the path in direction, doesn't belong to the path, or belongs to the path and agrees with the path in direction. The oriented path $\left\{e_{j_{1}}, \ldots, e_{j_{k}}\right\}$ is an oriented cycle if each of the two ends of any edge $e_{j_{i}}$ of the path is connected with another edge of the path. For the example of Figure B.1, the incidence vector of the cycle $\left\{e_{3}, e_{2}, e_{5}, e_{6}\right\}$ is $x=(0,1,1,0,1,1)^{T}$, and the incidence of the cycle $\left\{e_{1}, e_{4}, e_{6}, e_{3}\right\}$ is $x=(-1,0,1,-1,0,1)^{T}$.

Lemma B.1. (See Bapat [3], p. 57) If $x$ is the incidence vector of a cycle $\left\{e_{j_{1}}, \ldots, e_{j_{k}}\right\}$, then $Q x=0$.

Lemma B.2. (See Bapat [3], Lem. 2.2) If $Q$ is an incidence matrix of a connected graph, then $\operatorname{rank} Q=n-1$.

Rank-nullity theorem. (See e.g. Friedberg et al. [20], Thm. 2.3) Let $V$ and $W$ be vector spaces, and let $T: V \mapsto W$ be linear. If $V$ is finite-dimensional, then

$$
\operatorname{dim} \operatorname{Ker} T+\operatorname{rank} T=\operatorname{dim} V .
$$

Rockafellar ([50], Cor. 23.8.1). Let $C_{1}, \ldots, C_{m}$ be convex sets in $\mathbb{R}^{n}$ whose relative interiors have a point in common. Then the normal cone to $C_{1} \cap \ldots \cap C_{m}$ at any given point $x$ is $K_{1}+\ldots+K_{m}$, where $K_{i}$ is the normal cone to $C_{i}$ at $x$. If certain of the sets, say $C_{1}, \ldots, C_{k}$, are polyhedral, the conclusion holds if merely the sets $C_{1}, \ldots, C_{k}$, ri $C_{k+1}, \ldots$, ri $C_{m}$ have a point in common.

Let $\langle\cdot, \cdot\rangle$ be a standard scalar product in $\mathbb{R}^{n}$ and let $A$ be an $n \times n$-invertible matrix. We remind the reader that a finite-dimensional vector space $X=\mathbb{R}^{n}$ equipped with the norm $\|x\|=\sqrt{\langle x, A x\rangle}$ is an inner product space (see Narici-Beckenstein [46], Sect. 1.7.1).

Corollary of Theorems 16.1.4 and 16.1.5 from Narici-Beckenstein [46]. If $X$ is an inner product space, then, for any nonzero $x, y \in X,\|x+y\|=\|x\|+\|y\|$ implies that there exists $\alpha>0$ such that $y=\alpha x$. 


\section{Appendix C. Proof of Theorem 4.6}

Massera-Krejci Theorem for sweeping processes with a moving set of the form $C(t)=\cap_{j=1}^{M}\left(C_{j}+c_{j}(t)\right)$. We prove that every solution $x$ of sweeping process (4.1), that is defined on $[0, \infty)$, satisfies

$$
\lim _{t \rightarrow \infty}\left\|x(t)-x^{*}(t)\right\|_{0}=0,
$$

where $x^{*}$ is a $T$-periodic solution of (4.1).

Notice, that in case of $T$-periodic input the function $t \mapsto x(t+T)$ coincide with another solution of (4.1) originating from the point $x(T)$ at $t=0$. Due to monotonicity of $N_{C(t)}^{0}(x)$ in $x$ the distance $\|x(t+T)-x(t)\|_{0}$ is non-increasing (see e.g. Cor. 1 in [38]) and there exists

$$
r=\lim _{t \rightarrow \infty}\|x(t+T)-x(t)\|_{0} .
$$

Since $x([0, \infty))$ is precompact, there is a subsequence $\left\{n_{i}\right\}_{i \in \mathbb{N}} \subset \mathbb{N}$ and a point $x_{0}^{*}$ such that

$$
\lim _{i \rightarrow \infty}\left\|x\left(n_{i} T\right)-x_{0}^{*}\right\|_{0}=0 .
$$

Moreover, since each $x\left(n_{i} T\right) \in C\left(n_{i} T\right)=C(0)$ and $C(0)$ is closed we have $x_{0}^{*} \in C(0)$. Let $x^{*}$ be a solution of (4.1) with the initial condition $x^{*}(0)=x_{0}^{*}$. Consider the functions

$$
x_{i}(t)=x\left(t+n_{i} T\right), \quad i \in \mathbb{N} .
$$

Since $C(t)=C\left(n_{i} T+t\right)$, each function $x_{i}(t)$ is the solution of sweeping process (4.1) with the initial condition $x_{i}(0)=x\left(n_{i} T\right)$. The distance between solutions doesn't increase, so for any $t \geqslant 0$,

$$
0 \leqslant\left\|x_{i}(t)-x^{*}(t)\right\|_{0} \leqslant\left\|x\left(n_{i} T\right)-x_{0}^{*}\right\|_{0}
$$

and using (C.3) we obtain

$$
\lim _{i \rightarrow \infty}\left\|x\left(t+n_{i} T\right)-x^{*}(t)\right\|_{0}=\lim _{i \rightarrow \infty}\left\|x_{i}(t)-x^{*}(t)\right\|_{0}=0
$$

Let us prove that $x^{*}$ is $T$-periodic. Combining (C.5) and (C.2) we get

$$
r=\lim _{i \rightarrow \infty}\left\|x\left(t+n_{i} T+T\right)-x\left(t+n_{i} T\right)\right\|_{0}=\left\|x^{*}(t+T)-x^{*}(t)\right\|_{0} .
$$

Since $x^{*}$ and $t \mapsto x^{*}(t+T)$ are two solutions of sweeping process (4.1) with the constant distance $r$ between them, Lemma 4.5 yields

$$
\dot{x}^{*}(t)=\dot{x}^{*}(t+T), \quad t \geqslant 0
$$

Thus,

$$
x^{*}(\bar{n} T)-x_{0}^{*}=\int_{0}^{\bar{n} T} \dot{x}^{*}(t) \mathrm{d} t=\bar{n} \int_{0}^{T} \dot{x}^{*}(t) \mathrm{d} t=\bar{n}\left(x^{*}(T)-x_{0}^{*}\right), \quad \bar{n} \in \mathbb{N},
$$

and so $\left\|x^{*}(\bar{n} T)-x_{0}^{*}\right\|=\bar{n} r, \bar{n} \in \mathbb{N}$. Since $t \mapsto x^{*}(t)$ is bounded, the latter is possible only when $r=0$, i.e. when $x^{*}$ is $T$-periodic. 


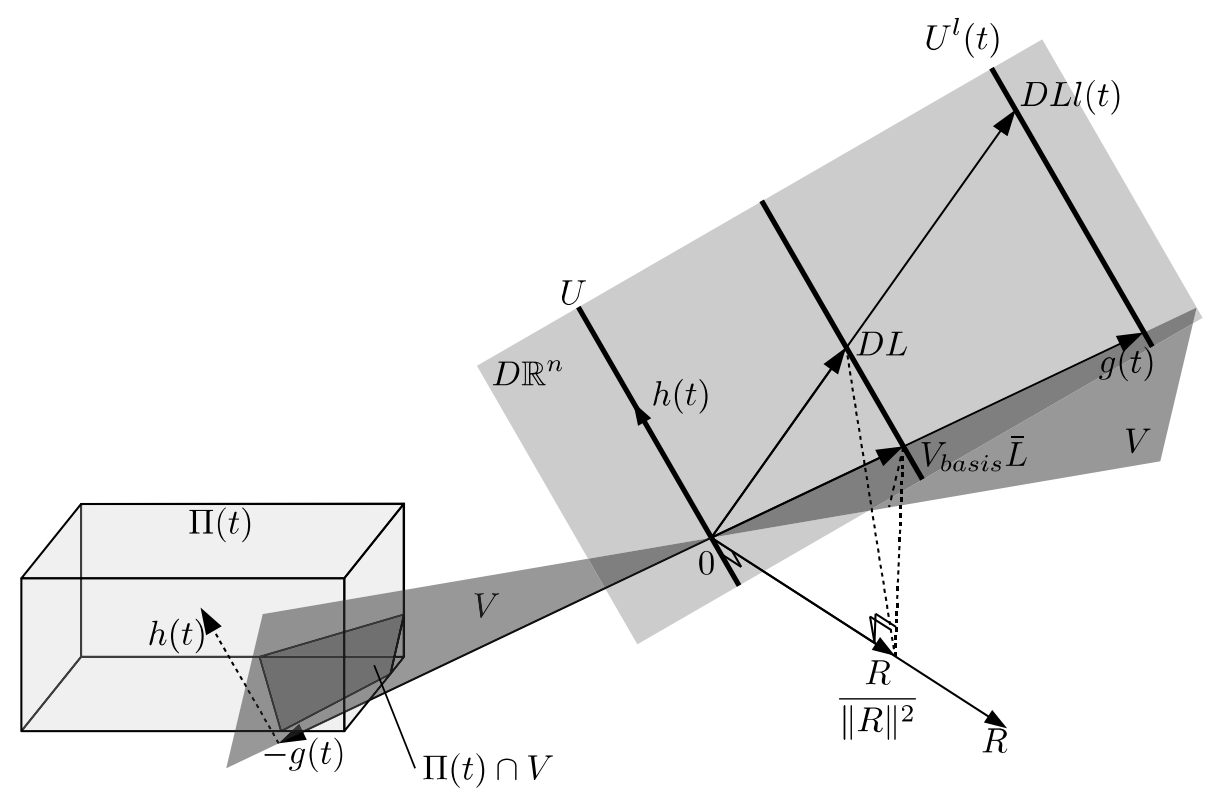

Figure D.1. The structure of the configuration space $\mathbb{R}^{m}$ when $q=1, \operatorname{dim} U=1, \operatorname{dim} V=2$. The right side of the figure shows how the vector $g(t)$ is obtained and the left side shows how the moving set $\Pi(t) \cap V$ is defined.

Now we finally prove (C.1). For each $t \geqslant 0$, we denote by $j(t)$ the maximal $j \in \mathbb{N}$ such that $n_{j(t)} T \leqslant t$. Then, letting $\tau(t)=t-n_{j(t)} T$, we use $T$-periodicity of $x^{*}$ and the property (C.5) to conclude

$$
\begin{aligned}
\lim _{t \rightarrow \infty}\left\|x(t)-x^{*}(t)\right\|_{0} & =\lim _{t \rightarrow \infty}\left\|x\left(\tau(t)+n_{j(t)} T\right)-x^{*}\left(\tau(t)+n_{j(t)} T\right)\right\|_{0} \leqslant \lim _{t \rightarrow \infty}\left\|x\left(n_{j(t)} T\right)-x^{*}\left(n_{j(t)} T\right)\right\|_{0} \\
& =\lim _{t \rightarrow \infty}\left\|x\left(n_{j(t)} T\right)-x^{*}(0)\right\|_{0}=\lim _{i \rightarrow \infty}\left\|x\left(n_{i} T\right)-x^{*}(0)\right\|_{0}=0 .
\end{aligned}
$$

The proof of the theorem is complete.

Similar to Theorem 4.6 results are obtained in Henriquez [25] (extension to Banach spaces) and in Kamenskii et al. [33] (extension to almost periodic solutions).

\section{Appendix D. Structure of the Configuration space}

To illustrate the structure of the configuration space $\mathbb{R}^{m}$ and construction of the variables used in Theorem 3.1 we can plot a 3D diagram (Fig. D.1) of a hypothetical situation when $m=3, q=1, \operatorname{rank} D=2$ whithout a connection to any particular network of springs. In such a case we have $\operatorname{dim} U=1, \operatorname{dim} V=2$. Since $q=1$ the matrices $R$ and $L$ are single column-vectors and we illustrate the condition (3.2) on $L$ by showing that

$$
\operatorname{proj}_{R}(D L)=\frac{R}{\|R\|}\left\langle\frac{R}{\|R\|}, D L\right\rangle=\frac{R}{\|R\|^{2}} R^{T} D L=\frac{R}{\|R\|^{2}}
$$

\section{COMPLIANCE WITH ETHICAL STANDARDS}

Conflict of Interest: The authors have no conflict of interest. 


\section{REFERENCES}

[1] S. Adly, M. Ait Mansour and L. Scrimali, Sensitivity analysis of solutions to a class of quasi-variational inequalities. Boll. Unione Mat. Ital. Sez. B Artic. Ric. Mat. 8 (2005) 767-771.

[2] S. Adly, F. Nacry and L. Thibault, Discontinuous sweeping process with prox-regular sets. ESAIM: COCV 23 (2017) $1293-1329$.

[3] R.B. Bapat, Graphs and matrices. Universitext. Springer, London (2010) 171.

[4] J. Bastien, F. Bernardin and C.-H. Lamarque, Non-smooth deterministic or stochastic discrete dynamical systems. Applications to models with friction or impact. Mechanical Engineering and Solid Mechanics Series. John Wiley \& Sons, Inc., Hoboken, NJ (2013) 496.

[5] T.R. Bieler, N.T. Wright, F. Pourboghrat, C. Compton, K.T. Hartwig, D. Baars, A. Zamiri, S. Chandrasekaran, P. Darbandi, H. Jiang, E. Skoug, S. Balachandran, G.E. Ice and W. Liu, Physical and mechanical metallurgy of high purity Nb for accelerator cavities. Phys. Rev. Spec. Top. 13 (2010) 031002.

[6] I. Blechman, Paradox of fatigue of perfect soft metals in terms of micro plasticity and damage. Int. J. Fatigue 120 (2019) 353-375.

[7] D. Bremner, K. Fukuda and A. Marzetta, Primal-dual methods for vertex and facet enumeration. ACM Symposium on Computational Geometry (Nice, 1997). Discr. Comput. Geom. 20 (1998) 333-357.

[8] B. Brogliato, Absolute stability and the Lagrange-Dirichlet theorem with monotone multivalued mappings. Syst. Control Lett. 51 (2004) 343-353.

[9] B. Brogliato, Nonsmooth mechanics. Models, dynamics and control. 3rd edn. Communications and Control Engineering Series. Springer, Berlin (2016).

[10] B. Brogliato and W.P.M.H. Heemels, Observer Design for Lur'e Systems With Multivalued Mappings: A Passivity Approach. IEEE Trans. Auto. Control 54 (2009) 1996-2001.

[11] B. Brogliato and L. Thibault, Existence and uniqueness of solutions for non-autonomous complementarity dynamical systems. J. Convex Anal. 17 (2010) 961-990.

[12] M. Brokate and J. Sprekels, Hysteresis and Phase Transitions, Springer, Berlin (1996).

[13] G.A. Buxton, C.M. Care and D.J. Cleaver, A lattice spring model of heterogeneous materials with plasticity. Modell. Simul. Mater. Sci. Eng. 9 (2001) 485-497.

[14] R. Cang, Y. Xu, S. Chen, Y. Liu, Y. Jiao and M.Y. Ren, Microstructure representation and reconstruction of heterogeneous materials via deep belief network for computational material design. J. Mech. Des. 139 (2017) 071404.

[15] H. Chen, E. Lin and Y. Liu, A novel Volume-Compensated Particle method for 2D elasticity and plasticity analysis. Int. J. Solids Struct. 51 (2014) 1819-1833.

[16] G. Colombo, R. Henrion, N.D. Hoang and B.S. Mordukhovich, Optimal control of the sweeping process over polyhedral controlled sets. J. Differ. Equ. 260 (2016) 3397-3447.

[17] J.B. Conway, A Course in Functional Analysis. 2nd edn. Springer, Berlin (1997).

[18] V. Crismale, Globally stable quasistatic evolution for a coupled elastoplastic-damage model. ESAIM: COCV 22 (2016) 883-912.

[19] C.O. Frederick and P.J. Armstrong, Convergent internal stresses and steady cyclic states of stress. J. Strain Anal. 1 (1966) $154-159$.

[20] S.H. Friedberg, A.J. Insel and L.E. Spence, Linear Algebra, 4th edn. Prentice-Hall of India, New Delhi (2004).

[21] G. Garcea and L. Leonetti, A unified mathematical programming formulation of strain driven and interior point algorithms for shakedown and limit analysis. Internat. J. Numer. Methods Eng. 88 (2011) 1085-1111.

[22] W. Grzesikiewicz, A. Wakulicz and A. Zbiciak, Mathematical modelling of rate-independent pseudoelastic SMA material. Int. J. Non-Linear Mech. 46 (2011) 870-876.

[23] W. Han and B.D. Reddy, Plasticity. Mathematical theory and numerical analysis. 2nd edn. Interdisciplinary Applied Mathematics, 9. Springer, New York (2013).

[24] M. Heitzer, G. Pop and M. Staat, Basis reduction for the shakedown problem for bounded kinematic hardening material. J. Global Optim. 17 (2000) 185-200.

[25] H.R. Henriquez, M. Pierri and P. Taboas, On S-asymptotically $\omega$-periodic functions on Banach spaces and applications. J. Math. Anal. Appl. 343 (2008) 1119-1130.

[26] J.-B. Hiriart-Urruty and C. Lemaréchal, Fundamentals of convex analysis. Grundlehren Text Editions. Springer-Verlag, Berlin $(2001) \mathrm{x}+259$.

[27] D.W. Holmes, J.G. Loughran and H. Suehrcke, Constitutive model for large strain deformation of semicrystalline polymers. Mech. Time-Depend Mater. 10 (2006) 281-313.

[28] H. Hubel, Simplified Theory of Plastic Zones. Springer, Berlin (2015).

[29] A. Isidori, Nonlinear control systems. An introduction. 2nd edn. Communications and Control Engineering Series. SpringerVerlag, Berlin (1989) xii+479.

[30] L. Jakabcin, A visco-elasto-plastic evolution model with regularized fracture. ESAIM: COCV 22 (2016) 148-168.

[31] M. Jirasek and Z.P. Bazant, Inelastic Analysis of Structures. Jhon Wiley \& Sons, London (2002).

[32] P. Jordan, A.E. Kerdok, R.D. Howe and S. Socrate, Identifying a Minimal Rheological Configuration: A Tool for Effective and Efficient Constitutive Modeling of Soft Tissues. J. Biomech. Eng. 133 (2011) 041006.

[33] M. Kamenskii, O. Makarenkov, L. Niwanthi Wadippuli and P. Raynaud de Fitte, Global stability of almost periodic solutions to monotone sweeping processes and their response to non-monotone perturbations. Nonlinear Anal. Hybrid Syst. 30 (2018) 213-224. 
[34] M.A. Krasnosel'skii, The operator of translation along the trajectories of differential equations. Translations of Mathematical Monographs, Vol. 19. Translated from the Russian by Scripta Technica. American Mathematical Society, Providence, R.I. (1968).

[35] M. Krasnosel'skii and A. Pokrovskii, Systems with Hysteresis. Springer, Berlin (1989).

[36] P. Krejci, Hysteresis, Convexity and Dissipation in Hyperbolic Equations. Gattotoscho, Tokyo (1996).

[37] P. Krejci and A. Vladimirov, Polyhedral sweeping processes with oblique reflection in the space of regulated functions. SetValued Anal. 11 (2003) 91-110.

[38] M. Kunze and M.D.P. Monteiro Marques, An introduction to Moreau's sweeping process. Impacts in mechanical systems (Grenoble, 1999), Vol. 551 of Lecture Notes in Physics. Springer, Berlin (2000) 1-60

[39] R.I. Leine and N. van de Wouw, Stability and convergence of mechanical systems with unilateral constraints, Lecture Notes in Applied and Computational Mechanics, 36. Springer-Verlag, Berlin (2008).

[40] R.I. Leine and N. van de Wouw, Uniform convergence of monotone measure differential inclusions: with application to the control of mechanical systems with unilateral constraints. Internat. J. Bifur. Chaos Appl. Sci. Eng. 18 (2008) 1435-1457.

[41] H.X. Li, Kinematic shakedown analysis under a general yield condition with non-associated plastic flow. Int. J. Mech. Sci. 52 (2010) $1-12$.

[42] C.W. Li, X. Tang, J.A. Munoz, J.B. Keith, S.J. Tracy, D.L. Abernathy and B. Fultz, Structural Relationship between Negative Thermal Expansion and Quartic Anharmonicity of Cubic ScF . Phys. Rev. Lett. 107 (2011) 195504.

[43] J.A.C. Martins, M.D.P Monteiro Marques and A. Petrov, On the stability of quasi-static paths for finite dimensional elasticplastic systems with hardening. ZAMM Z. Angew. Math. Mech. 87 (2007) 303-313.

[44] J.L. Massera, The existence of periodic solutions of systems of differential equations. Duke Math. J. 17 (1950) $457-475$.

[45] J.-J. Moreau, On unilateral constraints, friction and plasticity. New variational techniques in mathematical physics (Centro Internaz. Mat. Estivo (C.I.M.E.), II Ciclo, Bressanone, 1973). Edizioni Cremonese, Rome (1974) 171-32.

[46] L. Narici and E. Beckenstein, Topological vector spaces. 2nd edn. Vol. 296 of Pure and Applied Mathematics. CRC Press, Boca Raton, FL (2011).

[47] C. Polizzotto, Variational methods for the steady state response of elastic-plastic solids subjected to cyclic loads. Int. J. Solids Struct. 40 (2003) 2673-2697.

[48] A.R.S. Ponter and H. Chen, A minimum theorem for cyclic load in excess of shakedown, with application to the evaluation of a ratchet limit. Eur. J. Mech. A/Solids 20 (2001) 539-553.

[49] V. Recupero, BV continuous sweeping processes. J. Differ. Equ. 259 (2015) 4253-4272.

[50] R.T. Rockafellar, Convex analysis. Princeton Mathematical Series, No. 28. Princeton University Press, Princeton, N.J. (1970).

[51] J. Schwiedrzik, R. Raghavan, A. Burki, V. LeNader, U. Wolfram, J. Michler and P. Zysset, In situ micropillar compression reveals superior strength and ductility but an absence of damage in lamellar bone. Nat. Mater. 13 (2014) $740-747$.

[52] E. Svanidze, T. Besara, M.F. Ozaydin, C.S. Tiwary, J.K. Wang, S. Radhakrishnan, S. Mani, Y. Xin, K. Han, H. Liang, T. Siegrist, P. M. Ajayan and E. Morosan, High hardness in the biocompatible intermetallic compound $\beta-T i_{3} A u$. Sci. Adv. 2 (2016) e1600319.

[53] A. Visintin, Differential Models of Hysteresis. Springer, Berlin (1994).

[54] D. Weichert and G. Maier, Inelastic behavior of structures under variable repeated loads. Springer, New York (2002).

[55] J. Zhang, B. Koo, Y. Liu, J. Zou, A. Chattopadhyay and L. Dai, A novel statistical spring-bead based network model for self-sensing smart polymer materials. Smart Mater. Struct. 24 (2015) 085022.

[56] N. Zouain and R. SantAnna, Computational formulation for the asymptotic response of elastoplastic solids under cyclic loads. Eur. J. Mech. A/Solids 61 (2017) 267-278. 